\title{
La gestión del resultado: una aproximación empírica en el sector hotelero mediante ajustes por devengo
}

\author{
Laura Parte Esteban \\ Universidad Nacional de Educación a Distancia (UNED) \\ Facultad de Ciencias Económicas y Empresariales \\ Dpto. Economía de la Empresa y Contabilidad \\ Paseo Senda del Rey, 11 \\ 28040 Madrid \\ Tel: $91 / 3988966$ \\ E-mail:lparte@cee.uned.es
}

\author{
María Jesús Such Devesa \\ Universidad de Alcalá \\ Facultad de Ciencias Económicas y Empresariales \\ Dpto. Estadística, Estructura Económica y Organización Económica Internacional \\ Antiguo Colegio de Mínimos \\ Plaza de la Victoria, 2 \\ 28802 Alcalá de Henares (Madrid) \\ Tel. y Fax: 918854201 \\ E-mail:mjesus.such@uah.es
}

\begin{abstract}
RESUMEN
Este trabajo examina la hipótesis de evitar pérdidas y descensos en resultados en una muestra de empresas hoteleras. En primer lugar, se plantean los histogramas de frecuencias para observar la existencia de una discontinuidad en las series de resultados. En segundo lugar, se investiga si la discontinuidad observada en la representación gráfica de las series de resultados se puede atribuir a los ajustes por devengo discrecionales. La evidencia empírica muestra una discontinuidad gráfica y estadísticamente
\end{abstract}


significativa en el punto de cero beneficios. La comparación de los resultados declarados con los resultados pre-manipulación no permite rechazar la hipótesis de que los administradores se muestren indiferentes en la publicación de pequeños beneficios y pequeños incrementos en resultados.

Palabras clave: ajustes por devengo, gestión del resultado, histogramas de frecuencias, sector hotelero.

Clasificación JEL: M41, M21.

\begin{abstract}
This paper focuses on earnings benchmarks (avoiding earnings and decreases in earnings hypothesis) using a sample of Spanish hotel firms. First, we examine whether there is a discontinuity at zero point in the earnings distribution. Second, we investigate whether such a kink was driven by managers exploiting their discretion in accruals to avoid reporting losses and decreases in earnings. Empirical evidence shows a discontinuity in earnings distribution around zero point and statistically significant. The comparison between observed earnings and pre-managed earnings points out that managers are not indifferent to facing earnings figures.

Keywords: discretionary accruals, earnings distribution, earnings benchmarks, hotel industry.
\end{abstract}

JEL Classification: M41, M21.

\title{
1. INTRODUCCIÓN
}

El resultado contable es considerado una de las variables primordiales obtenidas de los estados financieros (Lev 1989). La cifra del resultado se usa como un indicador clave de la gestión realizada en el ejercicio por lo que los directivos no suelen ser indiferentes ante la publicación de cualquier nivel y variación de resultados. La importancia que se le ha atribuido al resultado contable a lo largo del tiempo y la asunción de que los inversores tienen en cuenta en la toma de decisiones puntos de referencia sencillos como puede ser observar resultados positivos, incrementos en resultados y sorpresas positivas, hace que los directivos traten de alcanzar estos umbrales de rentabilidad.

De esta manera, los estudios empíricos documentan que los administradores de las compañías son capaces de alterar el resultado en cualquiera de sus manifestaciones: el 
alisamiento del beneficio; el apuntamiento de la tendencia o depresión del resultado (taking a big bath), cuando la situación de la empresa lo requiere; o el logro de ciertos umbrales de rentabilidad que se consideran puntos críticos, como evitar pérdidas, descensos en resultados y sorpresas negativas en resultados, etc. En este trabajo nos centramos en la hipótesis de evitar pérdidas y descensos en resultados en una muestra de empresas hoteleras españolas.

Los estudios seminales de Hayn (1995) y Burgstahler y Dichev (1997) ponen de manifiesto la existencia de una discontinuidad en torno a cero beneficios y cero cambios en resultados. Burgstahler y Dichev (1997) observan, en la representación gráfica de los resultados, una baja densidad de observaciones en los intervalos inmediatamente anteriores al beneficio cero y una elevada densidad de observaciones en los intervalos inmediatamente posteriores al beneficio cero. Estos autores consideran que esta evidencia es fruto de una gestión intencionada por parte de los administradores de las compañías con la finalidad de alcanzar los umbrales de rentabilidad que se consideran críticos: evitar declarar pérdidas y descensos en resultados. Degeorge y otros (1999) añaden a los anteriores puntos críticos uno más: evitar sorpresas negativas en resultados.

Las ideas de estos autores, esto es, la prediposición gerencial a alterar el resultado alrededor de tres puntos de referencia que se consideran críticos, se ha demostrado satisfactoriamente en numerosos trabajos posteriores utilizando la metodología de los histogramas de frecuencias (Holland y Ramsay 2003) o bien mediante la metodología de los ajustes por devengo (Gill e Illueca 2003, Marquardt y Wiedman 2004) o ambas (Beatty y otros 2002, Beaver y otros 2003, Dechow y otros 2003, Thomas y otros 2004, Gallén y Giner 2005, Leone y Van Horn 2005 y Coulton y otros 2005, entre otros).

En este trabajo se utilizan las dos metodologías líderes en la detección de la gestión del resultado. Por un lado, los histogramas de frecuencias nos advierten de la existencia de una discontinuidad en el punto de cero beneficios. Por otro lado, los tres modelos de ajustes por devengo empleados nos permiten detectar la predisposición gerencial a utilizar los ajustes discrecionales como herramienta de manipulación de resultados.

Buena parte de los estudios empíricos que detectan prácticas de gestión del resultado utilizan alguno de los modelos de ajustes por devengo propuestos en la literatura contable. En este trabajo hemos optado por aplicar tres modelos de ajustes por devengo comúnmente empleados en estudios con un perfil similar al nuestro. Cabe apuntar que la muestra con la que trabajamos va a condicionar los resultados obtenidos y es posible que las características que presenta (entre ellas la reducida dimensión de las empresas) se muestre a favor de que el uso de los ajustes por devengo no sea el único recurso en manos de la gerencia para lograr ciertos retoques en el resultado. 
Es preciso señalar que la metodología de los histogramas de frecuencias ha recibido sus críticas, al igual que el resto de metodologías que tratan de captar o aproximarse a la realidad empresarial. Los avances empíricos señalan la importancia de controlar ciertos factores como el denominador de los resultados, la amplitud de los intervalos, la propia distribución del resultado, etc. para no derivar en un sesgo a favor de la gestión del resultado cuando en realidad no existe (Dechow y otros 2003, Holland 2004, Durtschi y Easton 2005 y posteriormente, Coulton y otros 2005, Ayers y otros 2006, Beaver y otros 2007, Hann y Lu 2007, entre otros).

En este contexto, los trabajos de Jacob y Jorgensen (2007) y Kerstein y Rai (2007) replican el estudio inicial de Burgstahler y Dichev (1997) teniendo en cuenta las contradicciones observadas en los últimos trabajos sobre la metodología de los histogramas de frecuencias y la veracidad de las prácticas de manipulación del resultado (Holland 2004 y Durtschi y Easton 2005, entre otros). Ambos se muestran a favor de la gestión del resultado (igual que Burgstahler y Dichev 1997 y sus seguidores) para batir los umbrales de referencia, una vez controladas las posibles fisuras de la metodología de los histogramas de frecuencias.

En este artículo se examina la hipótesis de evitar pérdidas y descensos en resultados en el sector hotelero español. La predisposición gerencial a alterar los resultados en torno a dichos umbrales de rentabilidad se ha detectado en ciertos sectores de la economía española y bajo ciertas restricciones. Por ejemplo, en el sector eléctrico (Gill e Illueca 2003), en empresas cotizadas y no cotizadas (Gallén y Giner 2005) así como mediante la utilización de variables concretas como el impuesto sobre beneficios (Parte y otros 2007) o las partidas extraordinarias (Parte 2008). También se puede observar la posición de nuestro país en estudios que utilizan muestras que engloban un conjunto de países (Leuz y otros 2003, Kinnunen y Koskela 2003 y Coppens y Peek 2005, entre otros).

El análisis del sector hotelero español es relevante por varias razones: a) en primer lugar, por su importancia en la economía española que hace que algunos autores se refieran a él como la principal actividad en la economía española, b) en segundo lugar, por la escasez de estudios existentes en este sector dada la dificultad de obtener información financiera, el limitado número de compañías del sector que cotizan, así como por la información incompleta que se encuentra disponible en el caso de las empresas que reportan, y c) en tercer lugar, no se conoce hasta el momento ningún trabajo que explote la línea de investigación emprendida en este trabajo. Todo ello hace que cualquier aproximación empírica sea de gran utilidad para investigadores y académicos y además permita comprender mejor las pautas de comportamiento de las empresas que lo conforman. 
En este trabajo se examina la hipótesis de evitar pérdidas y descensos en resultados en el sector hotelero español. Para ello se han seleccionado 1.990 empresas por año (9.950 observaciones) correspondientes al período 1999-2003, obtenidas de la base de datos SABI. El estudio se divide en dos etapas. En primer lugar, se plantean los histogramas de frecuencias de los resultados para observar la existencia de una discontinuidad en las series de resultados. En un análisis de sensibilidad se lleva a cabo una serie de controles sobre la representación gráfica de las series de resultados como el denominador utilizado y la amplitud de los intervalos, tal y como indican los últimos trabajos publicados. En segundo lugar, se investiga si la discontinuidad observada en los histogramas se puede atribuir a los ajustes por devengo discrecionales. Para ello se emplean los modelos de Jones original (1991), Jones modificado (Dechow y otros 1995) y Kothari y otros (2005).

En líneas generales la evidencia encontrada muestra una discontinuidad significativa en la representación gráfica de niveles de resultados y cambios en resultados. Los controles llevados a cabo en un análisis de sensibilidad sobre el denominador utilizado y la amplitud de los intervalos parecen no ser los causantes de una discontinuidad artificial. La comparación de los resultados declarados con los pre-manipulación no permite rechazar la hipótesis de que los administradores se muestren indiferentes en la publicación de pequeños beneficios y pequeños incrementos en resultados frente a pérdidas o pequeños descensos en resultados. No obstante, la evidencia obtenida señala que hay que tener prudencia en la interpretación de los ajustes por devengo discrecionales como indicativo indiscutible de una manipulación de resultados.

Hay razones que pueden justificar la evidencia encontrada a favor de una gestión intencionada por parte de los administradores para provocar determinados efectos en la cifra de beneficios como es evitar declarar pérdidas y descensos en resultados. Por un lado, el sector hotelero español se encuentra en un marco de carácter aperturista y de globalización de las relaciones económicas donde la captación de capitales se convierte en un problema fundamental. Los argumentos anteriores pueden motivar a los administradores a llevar a cabo ciertos retoques en la información contenida en los estados contables que les permita el acceso a las fuentes de financiación necesarias y les facilite emprender políticas expansivas. Cabe apuntar que las empresas del sector hotelero son de dimensión reducida lo que dificulta la captación de capitales en mercados financieros y la deuda bancaria se convierte en su fuente de financiación principal.

Por otro lado, la atomización de las empresas que conforman el sector hotelero provoca que los controles sobre la información financiera sean escasos y lejos de ser comparables con empresas de mayor dimensión como sucede en el caso de las empre- 
sas auditadas. Por último, se puede señalar un límite al alza sin control de los resultados que vendría dado por la fiscalidad, y que se encuentra íntimamente relacionada con las políticas contables aplicadas. Desde nuestro punto de vista, quizás éste es uno de los factores que los empresarios — particularmente de las pymes - controlan de forma precisa para no perder ciertas ventajas en el pago del impuesto sobre beneficios y puede, sin lugar a dudas, fomentar prácticas de empeoramiento de resultados.

La estructura del trabajo es la siguiente. En el apartado segundo se lleva a cabo una revisión de la literatura. En el apartado tercero se describe la muestra utilizada, se plantea el estudio empírico y se analizan los resultados obtenidos. En el apartado cuarto se exponen las conclusiones.

\section{REVISIÓN DE LA LITERATURA}

\subsection{Los estudios de partida: evidencia obtenida}

El desarrollo de la hipótesis de pérdidas se atribuye al estudio de Hayn que en 1995 observó que la relación retornos-ganancias es mucho más débil para empresas con pérdidas que con beneficios. Posteriormente, Burgstahler y Dichev (1997) consideran que los gerentes tienen puntos de referencia o niveles frontera tan sencillos como conseguir un beneficio positivo o cruzar ese punto. Es decir, plantean dos hipótesis básicas que ayudan a explicar la alteración del resultado: a) evitar la obtención de pérdidas, lo que se traduce en la práctica en alcanzar al menos pequeños beneficios y b) evitar disminuciones en el resultado, lo que se traduce en la práctica en conseguir mejoras o pequeños incrementos en los resultados.

Burgstahler y Dichev (1997) consideran que bajo la hipótesis nula de no manipulación, la distribución del resultado presentará una forma más o menos alisada en torno a cero beneficios. Para comprobar sus predicciones de partida utilizan una muestra de empresas estadounidenses y observan, en la representación gráfica de los resultados, una discontinuidad en torno a cero beneficios (hipótesis de evitar pérdidas) y cero cambios en resultados (hipótesis de evitar descensos en resultados). Dicho con otras palabras, estos autores advierten en la representación gráfica de los resultados, una baja densidad de observaciones en los intervalos inmediatamente anteriores al beneficio cero y una elevada densidad de observaciones en los intervalos inmediatamente posteriores al beneficio cero, frente a lo esperado en condiciones normales.

Trabajos posteriores que evidencian la existencia de una discontinuidad en la representación gráfica de los resultados y la atribuyen a una gestión intencionada por parte 
de los administradores para evitar declarar pérdidas o/y descensos en resultados son por ejemplo, Beatty y otros (2002), Beaver y otros (2003), y Leone y Van Horn (2005) en EE.UU.; Gore y otros (2007) en Reino Unido; Holland y Ramsay (2003) en Australia; Thomas y otros (2004) y Suda y Shuto (2005) en Japón; Gill e Illueca (2003), Gallén y Giner (2005), Parte y otros (2007) y Parte (2008) en España.

El marco teórico que explica estas prácticas de gestión de resultados proviene de la Teoría de los Costes de Transacción (Transaction Costs Theory) y de la Teoría Prospectiva (Prospect Theory). La primera considera que: a) la cifra de resultados es relevante en las transacciones o acuerdos llevados a cabo entre la entidad y los distintos agentes relacionados con ella, y además para la evaluación de la información financiera, y b) para la toma de decisiones se toman puntos de referencia más o menos sencillos, uno de los cuales es el resultado contable (véase Coase 1937). La segunda señala que las motivaciones psicológicas del proceso humano se basan en distinguir entre lo positivo y lo negativo, lo cual resulta aplicable también en el campo de las decisiones económicas. De este modo puede pensarse que existe un punto de referencia que actúa como nivel frontera que, si la gerencia consigue traspasar, se tomaría como éxito, pero si no se alcanza se interpretaría como fracaso (véase Kahnemnan y Tversky 1979, Kahnemann y otros 1991, Tversky y Kahnemann 1991).

Asimismo, Fields y otros (2001) llevan a cabo una revisión de la literatura de las diferentes motivaciones que incentivan a los administradores a emprender prácticas de gestión del resultado y completan el marco teórico. Estos autores mencionan cuestiones relacionadas con los costes de agencia, información asimétrica y externalidades que afectan a las partes vinculadas en el devenir de las empresas. Healy y Wahlen (1999) agrupan en tres los incentivos que los gerentes tienen para desarrollar prácticas de gestión del resultado: motivaciones relacionadas con los costes contractuales, motivaciones políticas y gubernamentales y motivaciones relacionadas con la valoración de la empresa en el mercado.

La mayor parte de las evidencias empíricas que documentan estas prácticas de gestión del resultado provienen del mercado estadounidense aunque también se ponen de manifiesto en otros mercados. Holland y Ramsay (2003) emplean la metodología de Burgstahler y Dichev (1997) para una muestra de empresas australianas y encuentran una discontinuidad en el punto de cero beneficios, tanto en niveles como en cambios de resultados. Desde este ángulo, parece que el mercado japonés se comporta de forma similar al estadounidense en lo que respecta a sobrepasar con éxito los umbrales de rentabilidad referenciados. Así, se observa que la gerencia tiene fuertes motivaciones para utilizar la discreción contable que posee para evitar declarar pérdidas y descensos en resultados (Suda y Shuto 2005) o para conseguir los tres puntos de referencia (Tho- 
mas y otros 2004). Estos últimos encuentran que la alteración de los resultados es más intensa en las matrices de los grupos de empresas que en la información presentada por todo el grupo y, principalmente, se lleva a cabo a través de operaciones relacionadas con sus filiales.

Los estudios de Beatty y otros (2002), Beaver y otros (2003) y Leone y Van Horn (2005) se diferencian del resto en las muestras elegidas, sector bancario, sector asegurador y los hospitales sin ánimo de lucro, por orden. Aunque con perspectivas diferentes todos ellos demuestran las premisas de origen. Por ejemplo, Leone y Van Horn (2005) dadas las características específicas que identifican la muestra objeto de estudio (entidades sin fines lucrativos), parten de la creencia de que los administradores perseguirán objetivos de diferente envergadura y, por tanto, no existirá una motivación tan fuerte de dirigir las ganancias hacia los umbrales de referencia señalados por la literatura contable. En contra de lo esperado, los resultados indican que las ganancias juegan un importante rol en este tipo de organizaciones y son el medio de evaluar la gestión de la dirección incluso aunque la presión del factor mercado, lógicamente, se vea debilitada. Concretamente observan que los gerentes prefieren: a) alisar el beneficio, b) deprimir los resultados ante un cambio en la cúpula directiva, y c) evitar pequeñas pérdidas. Beatty y otros (2002) presentan evidencia consistente con el uso de la provisión de morosidad por parte de un conjunto de bancos comerciales cotizados para evitar declarar pequeñas disminuciones en sus beneficios.

Cabe señalar que los análisis comparativos de ámbito internacional ponen de manifiesto diferencias sustanciales en las prácticas de gestión del resultado dependiendo principalmente del mercado donde opera la empresa. En líneas generales los estudios empíricos apuntan a los países continentales como más manipuladores frente a los de origen anglosajón (Leuz y otros 2003 y Coppens y Peek 2005, entre otros). Una serie de variables como los controles de la información financiera, la naturaleza y protección de los inversores, factores sociales, culturales, etc., parecen explicar estas diferencias.

\subsection{Avances en la línea de investigación}

Algunos trabajos publicados desde mediados de la década del 2000 cuestionan que la discontinuidad observada en los histogramas sea consecuencia de una alteración en resultados, tal y como se ha venido demostrando en los estudios que siguen esta línea de investigación como por ejemplo Dechow y otros (2003), Holland (2004), Durtschi y Easton (2005) y Beaver y otros (2007). Dechow y otros (2003: 3) exami- 
nan los ajustes discrecionales de empresas que justamente sobrepasan el punto de referencia frente a las que justamente lo pierden para comprobar la existencia de una alteración del resultado para evitar pérdidas. Los resultados encontrados señalan que las empresas con pequeñas ganancias presentan ajustes por devengo positivos pero no son significativamente mayores que los de las empresas con pequeños beneficios como se esperaría ante una conducta de gestión del resultado. En la misma dirección caminan los trabajos de Coulton y otros (2005) para una muestra de empresas australianas y Ayers y otros (2006) para una muestra de empresas estadounidenses.

El problema fundamental de utilización de simples puntos de referencia como indicadores de una gestión de resultados deriva de que la discontinuidad observada en la representación de las series de resultados puede ser causada por factores independientes y ajenos a ella. Entre las principales debilidades que presenta la metodología de los histogramas de frecuencias destacan las siguientes:

a) sesgos en el denominador utilizado para transformar los resultados brutos en una variable relativa y por tanto, comparable empresa a empresa. Durtschi y Easton (2005) argumentan que las empresas que declaran pequeñas pérdidas (pérdidas definitivas) son valoradas en el mercado de distinta manera que las empresas que declaran pequeños beneficios (beneficios definitivos) provocando automáticamente una discontinuidad en el histograma del resultado. Dicho con otras palabras, las compañías que se sitúan a la izquierda del punto cero beneficios tienen distinto denominador (un menor valor de mercado) que las compañías situadas a la derecha del punto cero (que presentan un mayor valor de mercado). Incluso aunque las series de resultados presentaran una distribución alisada en torno a cero, el propio mecanismo de convertir dicho resultado en un ratio (ya sea mediante el valor de mercado, el activo o las ventas) desembocaría en una discontinuidad.

b) sesgos en la elección de la amplitud de los intervalos de frecuencia cuando se representan las series de resultados. La amplitud de los intervalos se encuentra directamente relacionada con la distribución representada en el histograma y con el test-estadístico que discrimina entre la significatividad y no significatividad de la discontinuidad. Por tanto, la amplitud de los intervalos es un punto crítico en la representación de la serie de resultados y se aconseja emplear distintas alternativas para contrastar los resultados (Holland 2004).

En este contexto, Jacob y Jorgensen (2007), Kerstein y Rai (2007) y Hann y Lu (2007) replican el estudio inicial de Burgstahler y Dichev (1997) teniendo en cuenta 
las contradicciones observadas en los últimos trabajos sobre la veracidad de estas prácticas (Holland 2004, Durtschi y Easton 2005). Todos ellos se muestran a favor de la gestión del resultado para batir los umbrales de referencia, una vez controlados los principales sesgos encontrados en la metodología de los histogramas de frecuencias.

En este trabajo, en primer lugar se examina si existe una discontinuidad en torno a cero beneficios (para evitar pérdidas) y cero incrementos (para evitar descensos en resultados) en una muestra española de empresas hoteleras. Siguiendo las indicaciones de los últimos trabajos publicados se lleva a cabo un control sobre el denominador de los resultados y la amplitud de los intervalos (véase el análisis de sensibilidad). Posteriormente, se analiza si la discontinuidad observada puede ser atribuida al poder discrecional que los administradores poseen sobre los ajustes por devengo discrecionales.

\section{MuESTRA, PLANTEAMIENTO EMPÍRICO Y RESULTADOS OBTENIDOS}

\subsection{Muestra empleada}

La fuente para la obtención de la información financiera procede de la base de datos SABI (Sistema de Análisis de Balances Ibéricos), elaborada por Bureau Van Dijk Electronic Publishing, S.A. e Informa, Información Económica, S.A. Se han elegido las cuentas no consolidadas de empresas que corresponden al epígrafe 551 de CNAE-93, o alternativamente, declaran en su objeto social estar desarrollando alguna actividad alojativa de carácter hotelero. De esta forma, las empresas que conforman nuestra muestra son 1.990 empresas por año (9.950 observaciones) pertenecientes al sector hotelero español para el período 1999-2003.

En el cuadro 1 se pone de manifiesto que en el sector analizado existe una mayor propensión a evitar declarar pérdidas (alrededor de un $80 \%$ ) frente a evitar declarar descensos (que supera en la mayoría de los casos el 45\%). Asimismo, en las tres primeras columnas se observa que alrededor de un $20 \%$ de las empresas presentan pérdidas en resultados ordinarios mientras que este porcentaje disminuye aproximadamente dos puntos en el resultado neto. La misma evidencia se pone de manifiesto en las tres últimas columnas relativas a descensos en resultados. 
CuAdro 1.-Estadística descriptiva

\begin{tabular}{|c|c|c|c|c|c|c|c|c|c|c|c|c|}
\hline \multirow{2}{*}{ Años } & \multicolumn{2}{|c|}{$\mathrm{RO}^{\mathrm{ATT}} \mathrm{t}_{\mathrm{t}-1}$} & \multicolumn{2}{|c|}{$\mathrm{RAI} / \mathrm{AT}_{\mathrm{t}-1}$} & \multicolumn{2}{|c|}{$\mathrm{RN} / \mathrm{AT}_{\mathrm{t}-1}$} & \multicolumn{2}{|c|}{$\Delta \mathrm{RO} / \mathrm{AT}_{\mathrm{t}-1}$} & \multicolumn{2}{|c|}{$\Delta \mathrm{RAI} / \mathrm{AT}_{\mathrm{t}-1}$} & \multicolumn{2}{|c|}{$\Delta \mathrm{RN} / \mathrm{AT}_{\mathrm{t}-1}$} \\
\hline & $\mathrm{n}$ & $\%$ & $\mathrm{n}$ & $\%$ & $\mathrm{n}$ & $\%$ & $\mathrm{n}$ & $\%$ & $\mathrm{n}$ & $\%$ & $\mathrm{n}$ & $\%$ \\
\hline 2003 & 525 & 0,264 & 449 & 0,226 & 451 & 0,227 & 1.112 & 0,559 & 1.083 & 0,544 & 1.085 & 0,545 \\
\hline 2002 & 433 & 0,218 & 391 & 0,196 & 395 & 0,198 & 1.106 & 0,556 & 1.094 & 0,550 & 1.103 & 0,554 \\
\hline 2001 & 357 & 0,179 & 316 & 0,159 & 321 & 0.161 & 1.070 & 0,538 & 1.045 & 0,525 & 1.050 & 0,528 \\
\hline 2000 & 337 & 0,169 & 296 & 0,149 & 295 & 0.148 & 890 & 0,447 & 859 & 0,432 & 895 & 0,450 \\
\hline Pool & 1.652 & 0,208 & 1.452 & 0,182 & 1.462 & 0,184 & 4.178 & 0,525 & 4.081 & 0,513 & 4.133 & 0,519 \\
\hline
\end{tabular}

Nota: $n$ es el número de empresas, \% indica el porcentaje de empresas que reportan pérdidas cada año (tres primeras columnas) y el porcentaje de empresas que reportan descensos en resultados cada año (últimas tres columnas). $\mathrm{RO}_{\mathrm{AT}}$ es el resultado ordinario dividido por el activo al inicio del año, RAI/AT ${ }_{\mathrm{t}-1}$ es el resultado antes de impuestos dividido por el activo al inicio del año, $\mathrm{RN} \mathrm{AT}_{\mathrm{t}-1}$ es el resultado neto dividido por el activo al inicio del año, $\Delta \mathrm{RO} / \mathrm{AT}_{\mathrm{t}-1}$ es la variación en el resultado ordinario divido por el activo al inicio del año, $\Delta \mathrm{RAI}_{/} \mathrm{AT}_{\mathrm{t}-1}$ es la variación en el resultado antes de impuestos dividido por el activo al inicio del año, $\Delta \mathrm{RN}$ es la variación en el resultado neto dividido por el activo al inicio del año. En el cálculo de cambios en resultados se han usado dos denominadores $\left[\left(\mathrm{RN}_{\mathrm{t}}-\mathrm{RN}_{\mathrm{t}-1}\right) / \mathrm{AT}_{\mathrm{t}-1}\right]$ y $\left[\left(\mathrm{RN}_{\mathrm{t}}-\mathrm{RN}_{\mathrm{t}-1}\right) / \mathrm{AT}_{\mathrm{t}-2}\right]$. Los resultados obtenidos son similares por lo que se ha elegido la primera fórmula para no perder las observaciones de dos períodos.

\subsection{Las distribuciones de frecuencias de los resultados}

El análisis empírico comienza con el planteamiento de los histogramas de frecuencias lo que nos permite observar la existencia de una discontinuidad alrededor del punto cero en las series de resultados (niveles y cambios). Los gráficos 1 muestran la representación de las distribuciones de niveles (primera columna) y cambios de los resultados (segunda columna) divididos por el activo total al principio del período ${ }^{1}$. Se han utilizado tres tipos de resultados: ordinarios, antes de impuestos y neto. Para el cálculo de la amplitud de los intervalos se ha empleado la fórmula de Degeorge y otros (1999): 2(q3-q1)/ $\mathrm{N}^{-1 / 3}$ (siendo q3 el tercer cuartil, q1 el primer cuartil y $\mathrm{n}$ el número de observaciones).

${ }^{1}$ La mayor parte de los estudios empíricos estadounidenses utilizan el valor de mercado como denominador de los resultados. En nuestro caso ha resultado imposible dado que solamente cotizan dos empresas de la muestra. 
Todos los gráficos muestran una discontinuidad en el punto de cero beneficios o cero cambios en resultados (en el mismo sentido Burgstahler y Dichev 1997, Degeorge y otros 1999, Beaver y otros 2003, Dechow y otros 2003, Gallén y Giner 2005 y Hann y Lu 2007, entre otros). La discontinuidad pone de manifiesto que hay una menor densidad de observaciones a la izquierda del cero frente a lo esperado en condiciones normales (asumiendo una distribución más o menos alisada en dicho punto) y una mayor densidad de observaciones a la derecha del cero frente a lo esperado en condiciones normales. No obstante, esta evidencia preliminar no se puede atribuir directamente a una gestión intencionada por parte de los administradores para evitar pérdidas y descensos en resultados puesto que los histogramas tienen que pasar los controles oportunos.

GRÁFICo 1.-Distribución del resultado ordinario, antes de impuestos y neto (niveles y cambios)

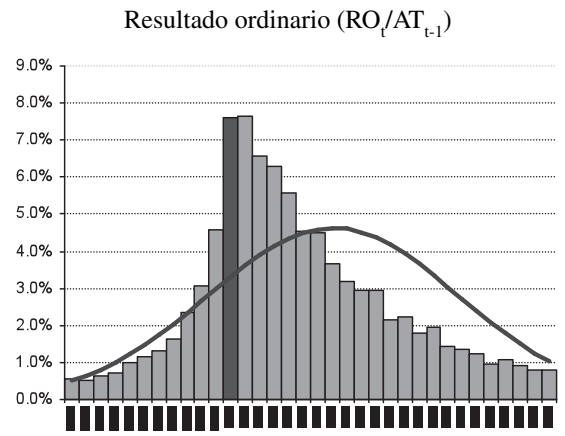

Resultado antes de impuestos $\left(\mathrm{RAI}_{\mathrm{t}} / \mathrm{AT}_{\mathrm{t}-1}\right)$

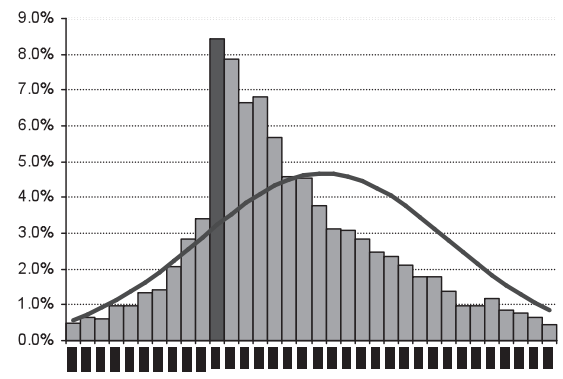

Cambios en resultados ordinarios $\left[\left(\mathrm{RO}_{\mathrm{t}}-\mathrm{RO}_{\mathrm{t}-1}\right) / \mathrm{AT}_{\mathrm{t}-1}\right]$

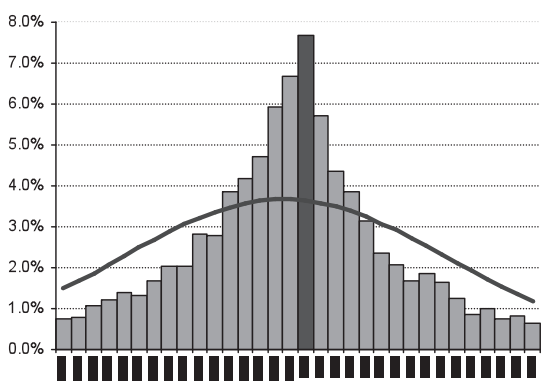

Cambios en resultados brutos $\left[\left(\mathrm{RAI}_{\mathrm{t}}-\mathrm{RAI}_{\mathrm{t}-1}\right) / \mathrm{A}_{\mathrm{t}-1}\right]$

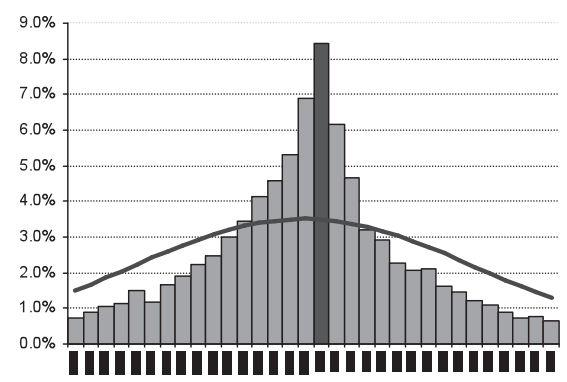


GRÁFICO 1.-Distribución del resultado ordinario, antes de impuestos y neto (niveles y cambios) (cont.)
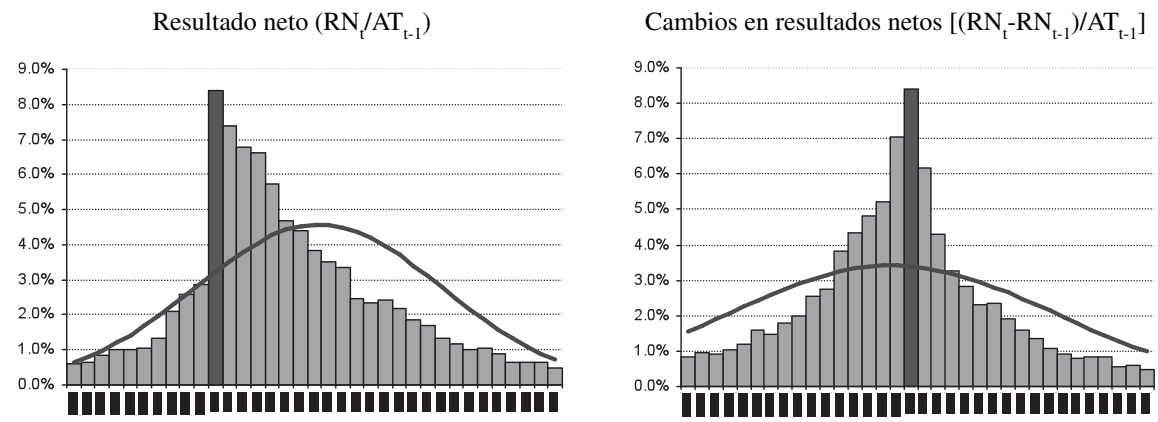

Nota: Los histogramas se han elaborado con datos pool a partir de 9.960 obs. Para el cálculo de la amplitud de los intervalos se ha seguido el criterio de Degeorge y otros (1999): (q3-q1)/ $\mathrm{N}^{-1 / 3}$ donde q3 es el cuartil tercero, q1 es el cuartil primero, $\mathrm{N}$ es el número de observaciones. Las amplitudes de los intervalos son de $(0,0114)$ para el resultado ordinario, $(0,0117)$ para el resultado antes de impuestos, $(0,0083)$ para el resultado neto, $(0,006)$ para el resultado ordinario, $(0,0062)$ para el resultado antes de impuestos y $(0,0047)$ para el resultado neto.

Las diferencias estandarizadas siguiendo las indicaciones de Burgstahler y Dichev (primer número) y Beaver y otros (segundo número) toman los valores de: RO (4,28 y 3,84), RAI (7,78 y 7,05), RN $(9,23$ y 8,41$), \Delta R O(4,24$ y 3,80$), \Delta R A I(5,22$ y 4,70$)$ y $\Delta R N(4,88$ y 4,39$)$.

La línea continua representa la distribución obtenida, sobre el supuesto de normalidad, dada la media de la población y la desviación típica de una muestra que concentra el $90 \%$ de los valores en torno a esta media, evitando así el efecto que sobre la dispersión tendrían los valores extremos. Todos los histogramas presentan asimetría y curtosis positivas. Las colas están truncadas. Para la definición de las variables véase la nota del cuadro 1.

GRÁFICo 2.—Distribución del resultado ordinario y neto por años

Resultado ordinario $\left(\mathrm{RO}_{\mathrm{t}} / \mathrm{AT}_{\mathrm{t}-1}\right)$

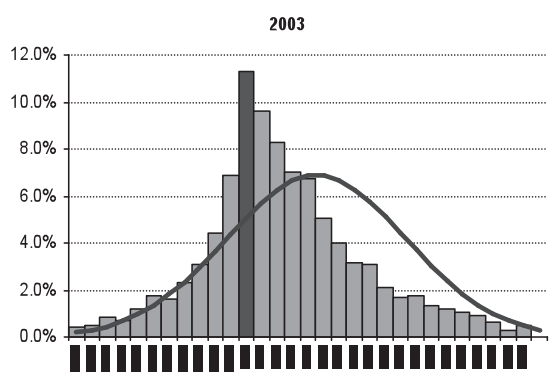

Resultado neto ( $\left.\mathrm{RN}_{\mathrm{t}} / \mathrm{AT}_{\mathrm{t}-1}\right)$

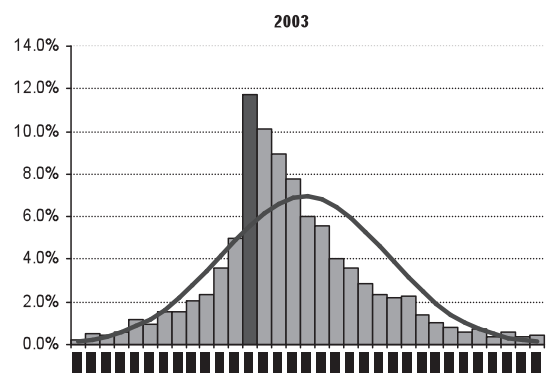


GRÁfICo 2.-Distribución del resultado ordinario y neto por años (cont.)
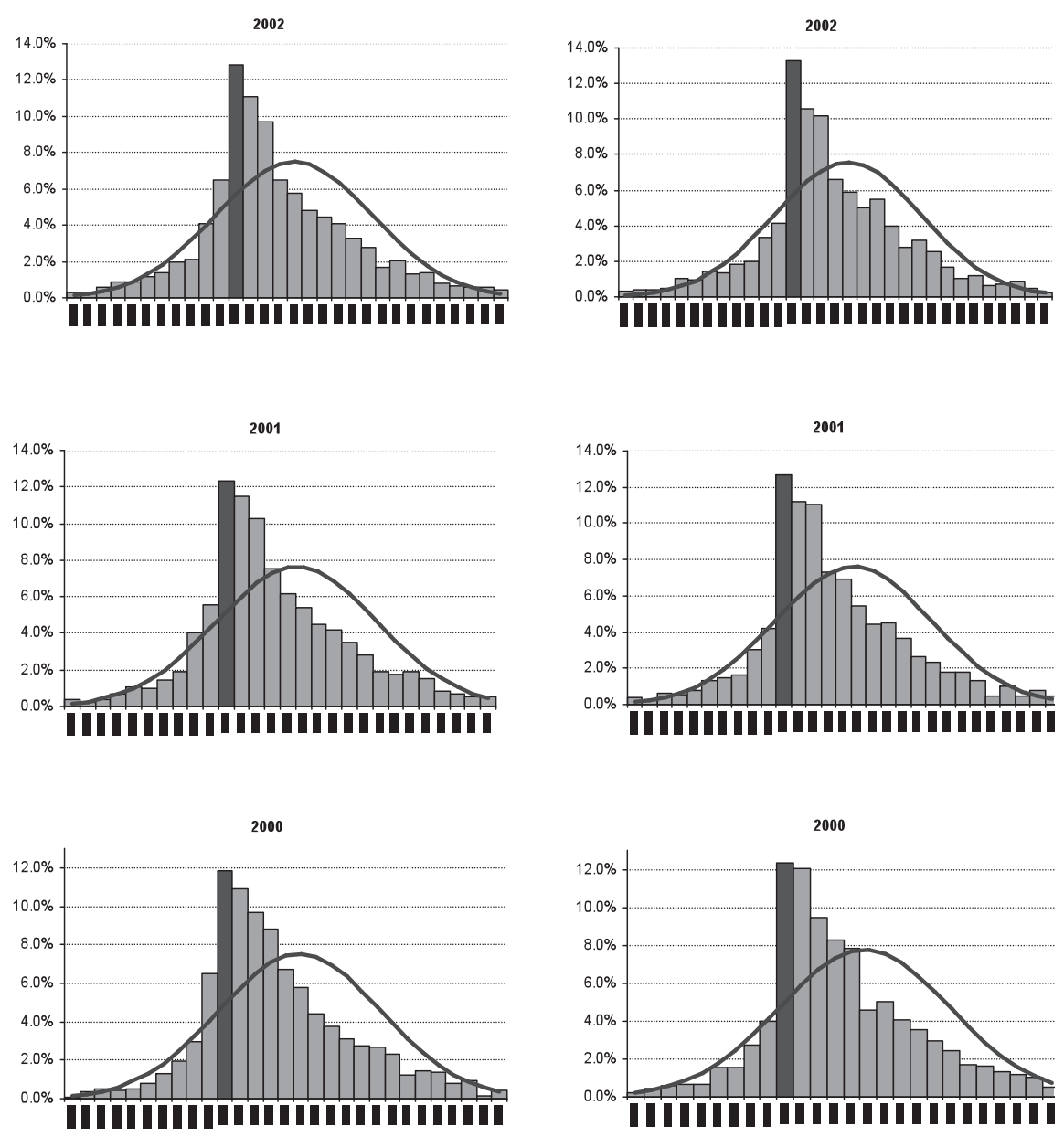

Nota: Para el cálculo de los histogramas véase la nota a pie de la página de los gráficos 1 . Las diferencias estandarizadas siguiendo las indicaciones de Burgstahler y Dichev (primer número) y Beaver y otros (segundo número) toman los valores de: RO (3,69 y 3,35), RN (5,06 y 4,61) para el año 2003 , RO $(4,74$ y 4,33), RN $(6,36$ y 6,91$)$ para el año 2002 , RO $(4,47$ y 4,07), RN $(5,87$ y 5,37$)$ para el año $2001, \mathrm{RO}(3,68$ y 3,65$)$, RN $(5,09$ y 4,65$)$ para el año 2000 . Todos los histogramas presentan asimetría y curtosis positivas. Las colas están truncadas. Similar evidencia se obtiene para el resultado antes de impuestos (RAI). La definición de las variables se encuentra en la nota del cuadro 1. 
En los gráficos 1 se puede observar que la discontinuidad en cambios (descensos) en resultados no es tan evidente como la de niveles (pérdidas) en resultados. En este sentido en el cuadro 1 se apreciaba que alrededor de un 55\% de las compañías del sector hotelero presentan descensos (más o menos acentuados) frente a un $45 \%$ que reportan aumentos en beneficios lo que a priori indica que la hipótesis de descensos de resultados tiene menor relevancia que la hipótesis de pérdidas. La diferencia estandarizada en el intervalo inmediatamente a la derecha del cero aumenta conforme nos situamos en niveles posteriores de resultados ${ }^{2}$. Concretamente, toma los siguientes valores RO ( 4,28 y 3,84$)$, RAI $(7,78$ y 7,05$), \mathrm{RN}(9,23$ y 8,41$), \Delta \mathrm{RO}(4,24$ y 3,80$), \Delta \mathrm{RAI}$ $(5,22$ y 4,70$)$ y $\Delta R N(4,88$ y 4,39$)$. La discontinuidad en el punto de cero beneficios se confirma estadísticamente $(p<0,05)$.

Los gráficos 2 muestran los histogramas de frecuencias calculados año a año. La evidencia encontrada con datos pool se repite ahora con datos anuales. Todos los gráficos muestran una discontinuidad en el punto de cero beneficios y que se acentúa a medida que escalamos posiciones de resultados. Lo mismo sucede con la diferencia estandarizada. El estadístico $t$ se muestra significativo en todos los casos. Similar evidencia se obtiene para cambios en resultados.

En definitiva, todos los histogramas revelan que la frecuencia de las observaciones a la izquierda del punto cero es menor que la esperada en condiciones normales mientras que a la derecha del punto cero es mayor que la esperada. En el siguiente apartado, se plantean los modelos de ajustes por devengo y se representa gráficamente la distribución del resultado pre-manipulación (antes de ajustes por devengo). La comparación de niveles y cambios en resultados declarados y pre-manipulación nos permite observar una serie de diferencias en las distribuciones de los resultados.

${ }^{2}$ La diferencia estandarizada se ha calculado siguiendo las indicaciones de Burgstahler y Dichev (1997: 102-103) y Beaver y otros (2007: 540, nota 12). La diferencia estandarizada viene dada por la diferencia entre el número actual de observaciones en un intervalo y el esperado, normalizada por la desviación típica de las diferencias. La fórmula propuesta por el primer autor es la siguiente: $\left[\mathrm{n}_{\mathrm{i}}-\left(\mathrm{n}_{\mathrm{i}-1}+\mathrm{n}_{\mathrm{i}+1}\right) / 2\right]\left[\mathrm{Np}_{\mathrm{i}}\left(1-\mathrm{p}_{\mathrm{i}}\right)+(1 / 4) \mathrm{N}\left(\mathrm{p}_{\mathrm{i}-1}+\mathrm{p}_{\mathrm{i}+1}\right)\right]^{1 / 2}$. Este test asume que la distribución de la diferencia estandarizada se aproxima a una normal de media 0 y desviación típica 1. Beaver y otros [2007: 540, nota 12] señalan que hay un error en la fórmula anterior, concretamente en la varianza que debería ser la siguiente: $\mathrm{Np}_{\mathrm{i}}\left(1-\mathrm{p}_{\mathrm{i}}\right)+(1 / 4) \mathrm{N}\left(\mathrm{p}_{\mathrm{i}-1}+\mathrm{p}_{\mathrm{i}+1}\right)\left(2-\mathrm{p}_{\mathrm{i}}-1-\mathrm{p}_{\mathrm{i}+1}\right)$. En cualquier caso, la corrección propuesta por estos autores disminuye sensiblemente los valores de la diferencia estandarizada aunque no altera los resultados. En este trabajo se han presentado los dos valores, el primero de ellos según las indicaciones de Burgstahler y Dichev y el segundo de ellos tiene en cuenta la modificación de Beaver y otros. 


\subsection{Los ajustes discrecionales y el logro de los puntos de referencia}

Buena parte de los estudios que se centran en detectar prácticas de gestión del resultado y más concretamente la hipótesis de evitar pérdidas y descensos en resultados, utiliza algún modelo de ajustes por devengo propuesto en la literatura. Por mencionar algunos, Beatty y otros (2002), Beaver y otros (2003), Dechow y otros (2003), Thomas y otros (2004), Gallén y Giner (2005), Leone y Van Horn (2005), Suda y Shuto (2005), Aryes y otros (2006), Gill e Illueca (2007) y Gore y otros (2007).

Entre los objetivos propuestos se encuentra: observar la existencia de una discontinuidad en torno a cero beneficios, comparar la distribución de resultados observados y pre-manipulación, observar la incidencia de los ajustes por devengo para alcanzar los puntos de referencia. La evidencia encontrada hasta el momento no es unidireccional. Así, Dechow y otros (2003), Coulton y otros (2005) y Gore y otros (2007), entre otros, no son capaces de demostrar que los ajustes discrecionales sean un instrumento en manos de los gerentes para lograr alcanzar los puntos de referencia mientras que la posición contraria puede verse en Beatty y otros (2002), Beaver y otros (2003), Gallén y Giner (2005), Leone y Van Horn (2005) y Suda y Shuto (2005), entre otros.

En esta línea de investigación es habitual utilizar modelos de ajustes por devengo agregados (McNichols y Wilson 1988) válidos para un amplio conjunto de muestras. Un elemento clave de los modelos de ajustes por devengo es desglosar la parte discrecional y no discrecional de los mismos. Los modelos de ajustes por devengo más populares en la investigación empírica son los de Jones original (1991) y Jones modificado (Dechow y otros 1995). Básicamente tratan de modelizar el componente normal o no discrecional en función de una serie de variables contables que asumen que el error de la regresión se corresponde con los ajustes motivados por actuaciones discrecionales de los directivos. Nos referimos a los ajustes anormales o discrecionales (ADD).

Los modelos de Jones original (1991) y Jones modificado (Dechow y otros 1995) asumen que el componente no discrecional de los ajustes totales (NDAC) es función del cambio de ventas (ventas ajustadas por las cuentas a cobrar) y el nivel del inmovilizado. En este trabajo, se ha estimado, además de estos dos modelos anteriores, la versión de Kothari y otros (2005) que considera necesario introducir una variable de eficiencia, la rentabilidad en los modelos anteriores.

Los primeros autores que estimaron los modelos por ajustes por devengo emplearon series temporales de cada empresa. Limitaciones derivadas del número de observaciones necesarias y la asunción de que los coeficientes son constantes en el tiempo relajaron la hipótesis de partida a favor de la utilización de datos de corte 
transversal para cada año y sector. La mayor parte de los estudios empíricos utiliza esta última alternativa puesto que se considera que produce mejores estimaciones de los coeficientes y los modelos se encuentran mejor especificados. A continuación se muestra las estimaciones sometidas a contrastación en nuestro trabajo, en versión cross-section:

$$
\begin{aligned}
& T A C_{i t} A T_{i t-1}=\alpha_{1}+\alpha_{2}\left[\Delta \operatorname{Vtas}_{i t} / A T_{i t-1}\right]+\alpha_{3}\left[\operatorname{Inmov}_{i t} / A T_{i t-1}\right]+\epsilon_{i t} \\
& T A C_{i t} A T_{t-1}=\alpha_{1}+\alpha_{2}\left[\left(\Delta \operatorname{Vtas}_{i t}-\Delta \operatorname{Clientes}_{i t}\right) / A T_{i t-1}\right]+\alpha_{3}\left[\operatorname{Inmov}_{i t} / A T_{i t-1}\right]+\epsilon_{i i} \\
& T A C_{i t} A T_{i t-1}=\alpha_{1}+\alpha_{2}\left[\Delta \operatorname{Vtas}_{i t} / A T_{i t-1}\right]+\alpha_{3}\left[\operatorname{Inmov}_{i t} / A T_{i t-1}\right]+\alpha_{4}\left[\operatorname{Recon} / A T_{i t-1}\right]+\epsilon_{i t}
\end{aligned}
$$

$$
\begin{aligned}
& W C A_{i t} A T_{i t-1}=\alpha_{1}+\alpha_{2}\left[\Delta \operatorname{Vtas}_{i t} / A T_{i t-1}\right]+\epsilon_{i i} \\
& W C A_{i t} A T_{t-1}=\alpha_{1}+\alpha_{2}\left[\left(\Delta \operatorname{Vtas}_{i t}-\Delta \operatorname{Clientes}_{i t}\right) / A T_{i t-1}\right]+\epsilon_{i i} \\
& W C A_{i t} A T_{i t-1}=\alpha_{1}+\alpha_{2}\left[\Delta \operatorname{Vtas}_{i} / A T_{i t-1}\right]+\alpha_{3}\left[\operatorname{Recon} / A T_{i t-1}\right]+\epsilon_{i t}
\end{aligned}
$$

Donde,

$\mathrm{TAC}_{\mathrm{it}}=$ ajustes por devengo totales de la empresa $i$ en el año $t$ (AActivo circulan-

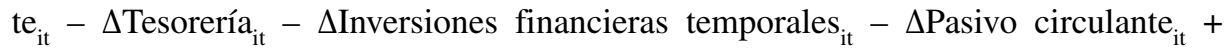

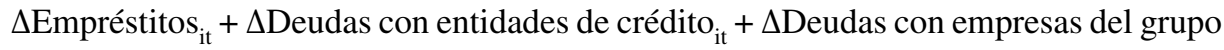
$\mathrm{y}$ asociadas $_{\text {it }}-$ Amortizaciones $_{\text {it }}-$ Provisiones $_{\text {it }}$ ) WCA $_{\text {it }}=$ ajustes por devengo a corto plazo de la empresa $i$ en el año $t$ (ajustes por devengo totales menos amortizaciones de la empresa $i$ para el año $t$ ); $\Delta \operatorname{Vtas}_{\text {it }}=$ variación en ventas para la empresa $\mathrm{i}$ en el período $t-1$ y $t$; Inmov $_{\text {it }}=$ inmovilizado de la $i$ en el año $t ;$ AT $_{\text {it- } 1}=$ activo total de la empresa $i$ en

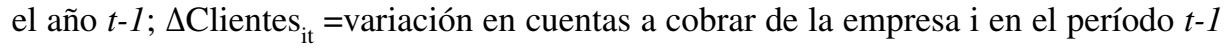
y $t ; \operatorname{Recon}_{\mathrm{it}}=$ rentabilidad económica de la empresa $i$ en el año $t$. Siguiendo las indicaciones de Kothari y otros (2005) se ha comprobado la especificación con dos variables Recon/AT ${ }_{i t}$ y Recon/A $\mathrm{it}_{-1}$, los resultados obtenidos son muy similares ${ }^{3}$.

${ }^{3}$ La mayor parte de las empresas presentan formatos abreviados de cuentas anuales por lo que en el cálculo de las variables se ha tenido que utilizar aproximaciones de lo que viene siendo habitual cuando se trabaja con formatos normales de cuentas anuales. 
La estimación de los ajustes no discrecionales de las ecuaciones previas (1-6), nos permite conocer, por diferencia, la magnitud de los ajustes discrecionales (ADD) de la empresa i para el año $t$. Concretamente se pueden calcular de la siguiente manera $\mathrm{ADD}_{i t}=T A C_{i t}\left(W C A_{i t}\right)-N D A C_{i t}$

El cuadro 2 contiene la estadística descriptiva de los ajustes por devengo para el período analizado y por modelo regresado. Se puede observar que tanto la media como la mediana son positivas con valores cercanos a cero. Los estadísticos $t$ para evaluar si el valor medio de los ajustes por devengo es distinto a cero no se muestran significativos en el período analizado. En este sentido, a priori no podemos observar si existe una manipulación al alza o a la baja de los resultados.

Cuadro 2.-Estadística descriptiva de los ajustes por devengo discrecionales (por modelo)

\begin{tabular}{|l|c|c|c|c|c|}
\hline & media & mediana & $\begin{array}{c}\text { Desv. } \\
\text { típica }\end{array}$ & $\max$ & $\min$ \\
\hline ADD1 (Jones original, largo) & 0,0000 & 0,0250 & 0,2953 & 1,8841 & $-3,7233$ \\
\hline ADD2 (Jones modificado, largo) & 0,0000 & 0,0273 & 0,2930 & 1,9092 & $-3,6855$ \\
\hline ADD3 (Kothari y otros, largo) & 0,0000 & 0,0256 & 0,2953 & 1,8827 & $-3,7252$ \\
\hline ADD4 (Jones original, corto) & 0,0000 & 0,0189 & 0,2928 & 1,5758 & $-3,6631$ \\
\hline ADD5 (Jones modificado, corto) & 0,0000 & 0,0198 & 0,2904 & 1,7571 & $-3,6033$ \\
\hline ADD6 (Kothari y otros, corto) & 0,0000 & 0,0206 & 0,2925 & 1,5930 & $-3,6574$ \\
\hline
\end{tabular}

Los gráficos 3 muestran la distribución del resultado pre-manipulación (niveles y resultados) dividido por el activo total al principio del año. En la elaboración de los gráficos se han seguido las mismas pautas que en los gráficos con datos observados ${ }^{4}$. Las representaciones gráficas presentan ahora una forma distinta a la evidencia obtenida hasta el momento. Particularmente, las distribuciones de los resultados se muestran alisadas en torno a cero y desaparece la irregularidad en dicho punto. La discontinuidad en el punto de cero beneficios y cero cambios no se presenta significativa $(p>0,05)$. Los mismos resultados se ponen de manifiesto si se representan las distribuciones de los resultados año a año (gráficos 4). En este contexto, se hace necesario un análisis adicional para verificar la evidencia obtenida.

${ }^{4}$ En un análisis de sensibilidad (apartado 3.4) se han comprobado los resultados usando distintas amplitudes en los intervalos (véase Holland 2004). Los resultados obtenidos se mantienen sin variaciones significativas. 
GRÁFICO 3.-Distribuciones de los resultados pre-manipulación (niveles y cambios)

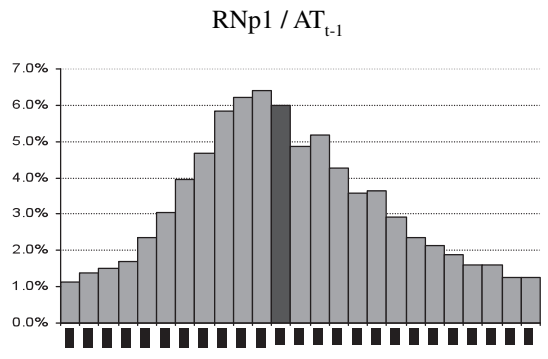

$\mathrm{RNp} 2 / \mathrm{AT}_{\mathrm{t}-1}$

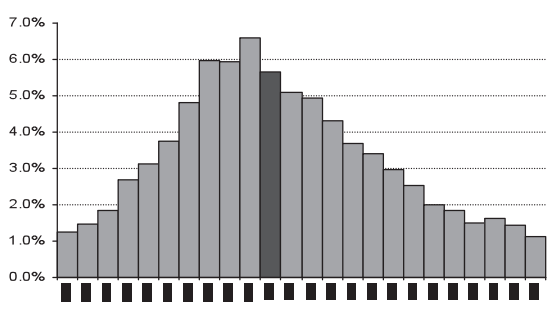

$\mathrm{RNp}_{3} / \mathrm{AT}_{\mathrm{t}-1}$

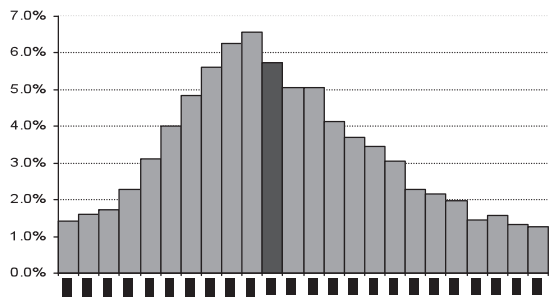

$\mathrm{RNp} 4 / \mathrm{AT}_{\mathrm{t}-1}$

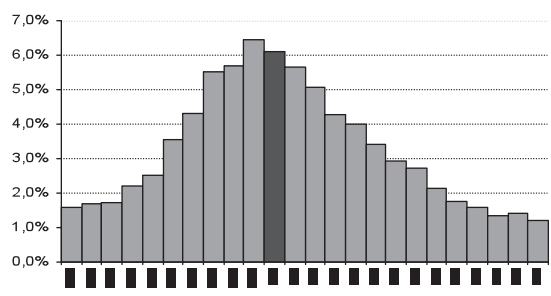

$\Delta \mathrm{RNp} 1 / \mathrm{AT}_{\mathrm{t}-1}$

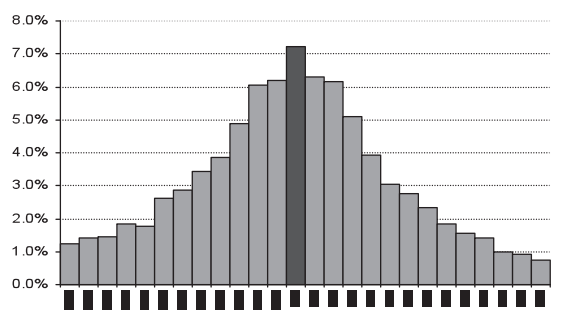

$\Delta \mathrm{RNp}^{2} / \mathrm{AT}_{\mathrm{t}-1}$

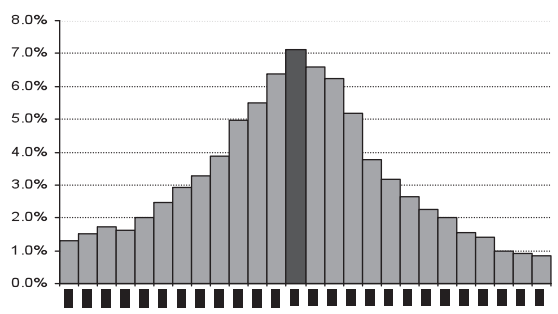

$\Delta \mathrm{RNp} 3 / \mathrm{AT}_{\mathrm{t}-1}$

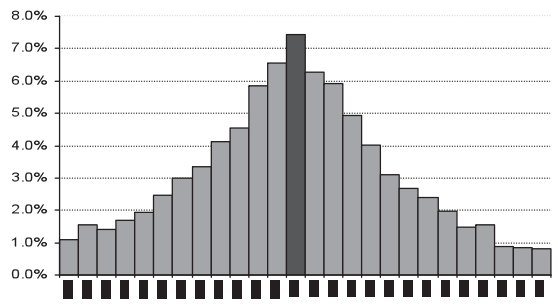

$\Delta \mathrm{RNp} 4 / \mathrm{AT}_{\mathrm{t}-1}$

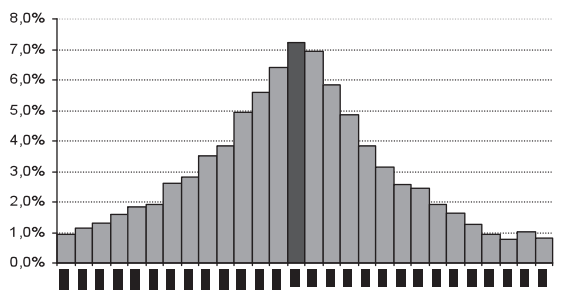

Nota: Los resultados pre-manipulación (RNp) se calculan como la diferencia entre el resultado observado y los ajustes discrecionales. RNp1 se obtiene del modelo de ajustes por devengo Jones original, RNp2 del modelo de Jones modificado (Dechow y otros 1995), RNp3 del modelo de Kothari y otros (2005), todos ellos a largo plazo. Similares resultados se obtienen cuando se calculan los ajustes a corto plazo. RNp4 es el modelo de Jones original a corto plazo. 
La amplitud de los intervalos se calcula de acuerdo con la fórmula de Degeorge y otros (1999) y toman los siguientes valores: $(0,022)$ para $R N p 1, R N p 2, R N p 3$ y $R N p 4$ y $(0,032)$ para $\Delta R N p 1$, $\Delta \mathrm{RNp} 2, \Delta \mathrm{RNp} 3$ y $\Delta \mathrm{RNp} 4$. Las diferencias estandarizadas en el intervalo inmediatamente a la derecha, siguiendo las indicaciones de Burgstahler y Dichev (primer número) y Beaver y otros (segundo número) toman los siguientes valores: RNp1 (1,08 y 0,96), RNp2 (0,62 y 0,54), RNp3 $(0,22$ y 0,19$), \operatorname{RNp} 4(0,19$ y 0,17$), \Delta R N p 1(2,46$ y 2,20$), \Delta \operatorname{RNp} 2(1,62$ y 1,45$), \Delta R N p 3(2,5$ у $2,23)$ y $\Delta$ RNp4 $(1,34$ y 1,19). El estadístico $t$ no se muestra significativo en ninguna distribución $(p>0,01)$. Todos los resultados se encuentran divididos por el Activo a principios del año. Las colas están truncadas.

GRÁFICO 4.-Distribuciones de los niveles de resultados netos pre-manipulación (RNp1) y cambios en resultados netos pre-manipulación $(\Delta \mathrm{RNp} 1)$. Modelo de Jones original, por años

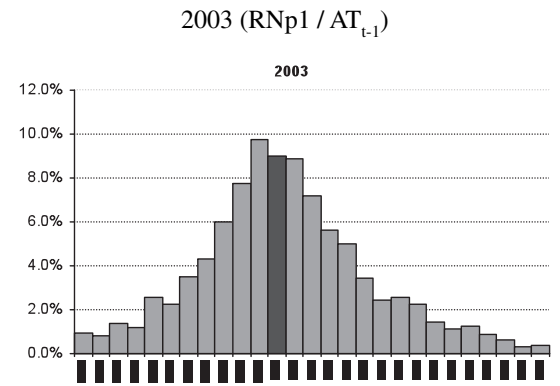

$2002\left(\mathrm{RNp}_{1} / \mathrm{AT}_{\mathrm{t}-1}\right)$

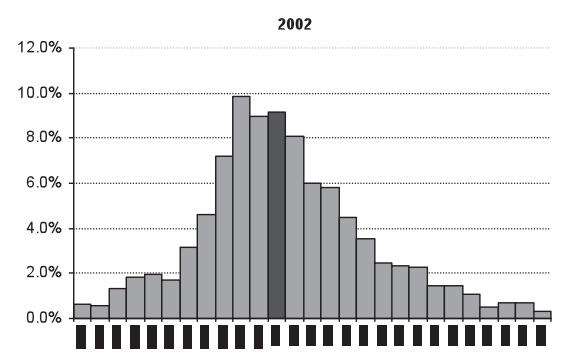

$2001\left(\mathrm{RNp}_{1} / \mathrm{AT}_{\mathrm{t}-1}\right)$

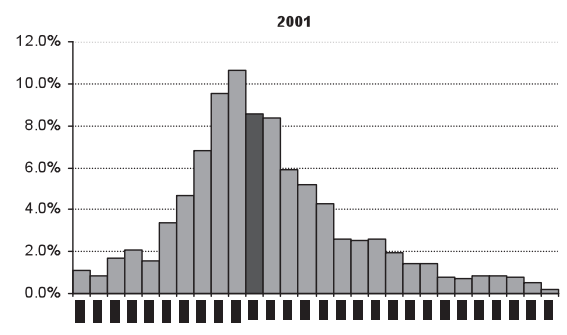

$2003\left(\Delta \mathrm{RNp} 1 / \mathrm{AT}_{\mathrm{t}-1}\right)$

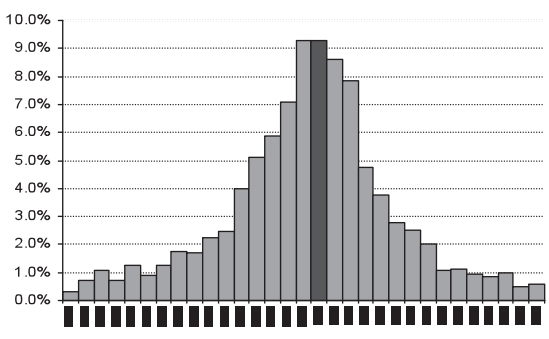

$2002\left(\Delta \mathrm{RNp} 1 / \mathrm{AT}_{\mathrm{t}-1}\right)$

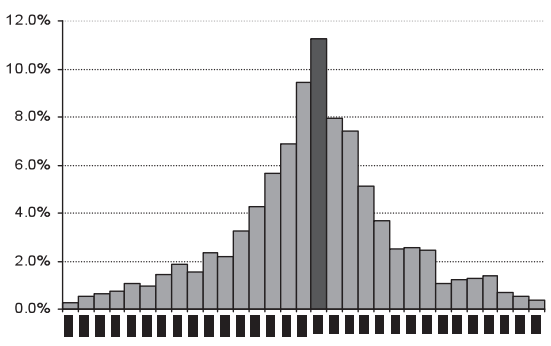

$2001\left(\Delta \mathrm{RNp}_{1} / \mathrm{AT}_{\mathrm{t}-1}\right)$

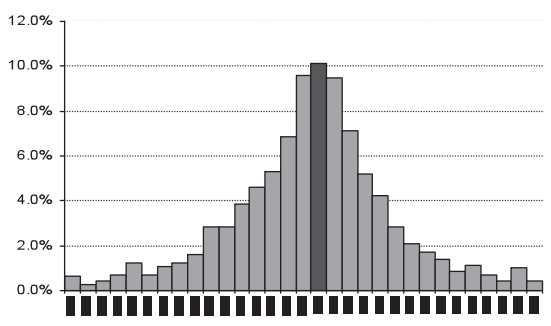


GRÁFICO 4.-Distribuciones de los niveles de resultados netos pre-manipulación (RNp1) y cambios en resultados netos pre-manipulación $(\Delta \mathrm{RNp} 1)$. Modelo de Jones original, por años (cont.)

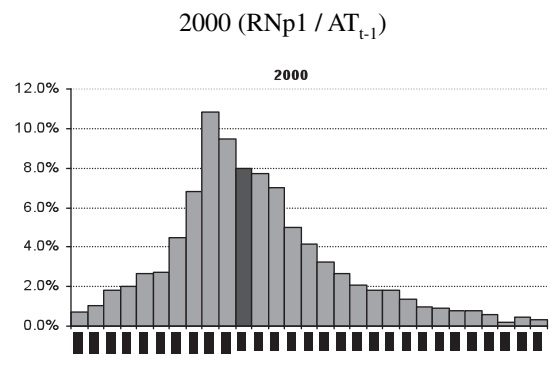

Nota: Para la definición de los resultados pre-manipulación (RNp) véase la nota a pie de página de los Gráficos 3. La amplitud de los intervalos se ha calculado de acuerdo con la fórmula de Degeorge y otros y toma los siguientes valores para RNpl (niveles de resultados): $(0,017)$ en $2003,(0,018)$ en $2002,(0,021)$ en 2001 y $(0,033)$ en el 2000 . La amplitud de los intervalos toma los siguientes valores para $\triangle \mathrm{RNp} 1$ (cambios de resultados): $(0,025)$ en 2003, $(0,026)$ en 2002 y $(0,036)$ en 2001. Las observaciones del primer año (el 2000) se han perdido al utilizar como denominador el activo del año previo. Las colas están truncadas. El estadístico $t$ no se muestra significativo en ningún período $(p>0,01)$.

Holland y Ramsay (2003: 41) señalan que la existencia de errores en la medición de los ajustes discrecionales es un problema fundamental en la investigación de la calidad del resultado. Estos autores advierten que estudios recientes tratan de evitar este inconveniente teniendo especial cuidado en el planteamiento de los modelos de ajustes por devengo, siendo habitual estimar en versión pool, año a año, transversal, etc. En nuestro estudio se han tenido en cuenta los problemas metodológicos en la determinación de los ajustes por devengo discrecionales y se han estimado todos los modelos año a año y en versión pool. Cabe recordar que la mayor parte de las empresas que conforma nuestro estudio son pymes y autores como Gill e Illueca (2007) advierten que, a pesar de estar sujetas a los mismos principios contables, la información financiera reportada por las empresas pequeñas se utiliza de distinto modo que la suministrada por las grandes compañías.

En el cuadro 3 se presenta un control de los ajustes por devengo para comprobar si son capaces de recoger las prácticas de gestión del resultado. Para ello se toman como base las distribuciones de los resultados pre-manipulación y los resultados observados calculadas con intervalos fijos de $0,01^{5}$. El panel A del cuadro 3 aparece la compara-

${ }^{5}$ La elección del intervalo de acuerdo con Burgstalher y Dichev (1997) se obtiene a través de la diferencia entre la frecuencia real y la esperada (sin manipulación) normalizada por la desviación típica de las diferencias. 
ción de los ajustes discrecionales de las empresas que justamente sobrepasan el umbral de rentabilidad crítico (zona de pequeños beneficios medida por los 3 intervalos inmediatamente a la derecha de cero) con el resto de empresas (muestra de control). Se han utilizado los tres modelos de ajustes por devengo estimados (véase el cuadro 3). De acuerdo con los argumentos de Burgstahler y Dichev (1997) y Dechow y otros (2003) se espera que los ajustes por devengo discrecionales sean significativamente más elevados en la zona crítica frente a la muestra de control, indicando, así, que son capaces de recoger la flexibilidad (discrecionalidad) contable.

La comparación de los ajustes por devengo en las empresas con pequeños beneficios respecto al resto de empresas (muestra de control) soporta la idea de que los ajustes por devengo son un instrumento de gestión de resultados. En el panel A del cuadro 3 se pone de manifiesto, en todos los modelos de ajustes por devengo, que las empresas que sobrepasan el punto de cero beneficios presentan ajustes por devengo más elevados que el resto de empresas y la diferencia en media y mediana alcanza la significatividad requerida estadísticamente. Puede observarse que la diferencia en los ajustes por devengo entre los dos grupos se refleja también en los ajustes totales y en los flujos de caja (en el mismo sentido Coulton y otros 2005: 565).

En el panel B (cuadro 3) se contrastan los ajustes por devengo de las empresas que justamente no alcanzan el punto cero (zona de pequeñas pérdidas, medida como los tres intervalos inmediatamente a la izquierda de cero) con respecto al resto de empresas (muestra de control). La evidencia camina en la misma dirección que en el panel anterior. Esto es, se pone de manifiesto que los ajustes por devengo (en cualquiera de los modelos utilizados) son estadísticamente diferentes en media y mediana en ambas segmentaciones y además son mayores en las empresas que presentan pequeñas pérdidas frente a la muestra de control.

CuAdro 3.-Control de los ajustes por devengo discrecionales (ADD)

\begin{tabular}{|c|c|c|c|c|c|c|c|c|c|c|}
\hline \multicolumn{7}{|c|}{ Panel A: Muestra total versus empresas con pequeños beneficios } \\
\hline & \multicolumn{9}{|c|}{ Muestra Total } & \multicolumn{3}{|c|}{ Pequeños beneficios $(0<\mathrm{RN}<0,03)$} & \multicolumn{3}{c|}{ Test-diferencias } \\
\hline Vbles & $\mathrm{N}$ & media & mediana & desv. & $\mathrm{N}$ & media & mediana & desv. & media & mediana \\
\hline ADT & 7.960 & $-0,0920$ & $-0,0643$ & 0,3049 & 2.139 & $-0,0679$ & $-0,0578$ & 0,2358 & 0,0001 & 0,0127 \\
\hline CFO & 7.960 & 0,1443 & 0,1027 & 0,3220 & 2.139 & 0,0836 & 0,0726 & 0,2531 & 0,0000 & 0,0000 \\
\hline ADD1 & 7.960 & 0,0000 & 0,0250 & 0,2953 & 2.139 & 0,0218 & 0,0325 & 0,2258 & 0,0002 & 0,0048 \\
\hline ADD2 & 7.960 & 0,0000 & 0,0273 & 0,2930 & 2.139 & 0,0228 & 0,0346 & 0,2233 & 0,0001 & 0,0019 \\
\hline ADD3 & 7.960 & 0,0000 & 0,0256 & 0,2953 & 2.139 & 0,0244 & 0,0355 & 0,2258 & 0,0000 & 0,0006 \\
\hline
\end{tabular}


CUADRo 3.-Control de los ajustes por devengo discrecionales (ADD) (cont.)

\begin{tabular}{|l|l|l|l|l|l|l|l|l|l|l|}
\hline ADD4 & 7.960 & 0,0000 & 0,0189 & 0,2928 & 2.139 & 0,0167 & 0,0223 & 0,2331 & 0,0056 & 0,1535 \\
\hline ADD5 & 7.960 & 0,0000 & 0,0198 & 0,2904 & 2.139 & 0,0174 & 0,0235 & 0,2307 & 0,0035 & 0,0938 \\
\hline ADD6 & 7.960 & 0,0000 & 0,0206 & 0,2925 & 2.139 & 0,0222 & 0,0281 & 0,2329 & 0,0002 & 0,0052 \\
\hline
\end{tabular}

Panel B: Muestra total versus empresas con pequeñas pérdidas

\begin{tabular}{|c|c|c|c|c|c|c|c|c|c|c|}
\hline & \multicolumn{4}{|c|}{ Muestra Total } & \multicolumn{3}{c|}{ Pequeñas pérdidas $(-0,03<\mathrm{RN}<0)$} & \multicolumn{3}{c|}{ Test-diferencias } \\
\hline Vbles & $\mathrm{N}$ & media & mediana & desv. & $\mathrm{N}$ & media & mediana & desv. & media & mediana \\
\hline ADT & 7.960 & $-0,0920$ & $-0,0643$ & 0,3049 & 666 & $-0,0620$ & $-0,0511$ & 0,2607 & 0,0786 & 0,0441 \\
\hline CF0 & 7.960 & 0,1443 & 0,1027 & 0,3220 & 666 & 0,0526 & 0,0402 & 0,2610 & 0,0000 & 0,0000 \\
\hline ADD1 & 7.960 & 0,0000 & 0,0250 & 0,2953 & 666 & 0,0331 & 0,0349 & 0,2439 & 0,0179 & 0,0367 \\
\hline ADD2 & 7.960 & 0,0000 & 0,0273 & 0,2930 & 666 & 0,0339 & 0,0378 & 0,2426 & 0,0135 & 0,0250 \\
\hline ADD3 & 7.960 & 0,0000 & 0,0256 & 0,2953 & 666 & 0,0374 & 0,0398 & 0,2440 & 0,0051 & 0,0052 \\
\hline ADD4 & 7.960 & 0,0000 & 0,0189 & 0,2928 & 666 & 0,0141 & 0,0214 & 0,2421 & 0,0125 & 0,0023 \\
\hline ADD5 & 7.960 & 0,0000 & 0,0198 & 0,2904 & 666 & 0,0141 & 0,0222 & 0,2408 & 0,0118 & 0,0012 \\
\hline ADD6 & 7.960 & 0,0000 & 0,0206 & 0,2925 & 666 & 0,0236 & 0,0310 & 0,2422 & 0,1494 & 0,0916 \\
\hline
\end{tabular}

\begin{tabular}{|c|c|c|c|c|c|c|c|c|c|c|}
\hline \multicolumn{2}{|c|}{ Panel C: Empresas con pequeñas pérdidas versus empresas con pequenos beneficios } \\
\hline & \multicolumn{3}{|c|}{ Pequeñas pérdidas $(-0,05<\mathrm{RN}<0)$} & \multicolumn{3}{|c|}{ Pequeños beneficios $(0<\mathrm{RN}<0,05)$} & \multicolumn{3}{c|}{ Test-diferencias } \\
\hline Vbles & $\mathrm{N}$ & media & mediana & desv. & $\mathrm{N}$ & media & mediana & desv. & media & mediana \\
\hline ADT & 875 & $-0,0780$ & $-0,0576$ & 0,2740 & 3.176 & $-0,0649$ & $-0,0556$ & 0,2242 & 0,1941 & 0,5780 \\
\hline CFO & 875 & 0,0591 & 0,0400 & 0,2737 & 3.176 & 0,0883 & 0,0780 & 0,2414 & 0,0043 & 0,0000 \\
\hline ADD1 & 875 & 0,0186 & 0,0317 & 0,2619 & 3.176 & 0,0249 & 0,0357 & 0,2155 & 0,5147 & 0,5656 \\
\hline ADD2 & 875 & 0,0187 & 0,0348 & 0,2607 & 3.176 & 0,0259 & 0,0371 & 0,2123 & 0,4515 & 0,5213 \\
\hline ADD3 & 875 & 0,0236 & 0,0364 & 0,2620 & 3.176 & 0,0268 & 0,0373 & 0,2155 & 0,7365 & 0,9691 \\
\hline ADD4 & 875 & 0,0013 & 0,0183 & 0,2620 & 3.176 & 0,0220 & 0,0246 & 0,2219 & 0,0330 & 0,0641 \\
\hline ADD5 & 875 & 0,0006 & 0,0194 & 0,2612 & 3.176 & 0,0228 & 0,0262 & 0,2189 & 0,0214 & 0,0514 \\
\hline ADD6 & 875 & 0,0124 & 0,0305 & 0,2621 & 3.176 & 0,0262 & 0,0295 & 0,2217 & 0,1563 & 0,6627 \\
\hline
\end{tabular}

Panel D: Empresas con pequeñas pérdidas versus empresas con elevadas pérdidas

\begin{tabular}{|c|c|c|c|c|c|c|c|c|c|c|}
\hline & \multicolumn{4}{|c|}{ Pequeñas pérdidas $(-0,03<\mathrm{RN}<0)$} & \multicolumn{4}{c|}{ Extremo $(\mathrm{RN}<-0,4)$} & \multicolumn{3}{c|}{ Test-diferencias } \\
\hline Vbles & $\mathrm{N}$ & media & mediana & desv. & $\mathrm{N}$ & media & mediana & desv. & media & mediana \\
\hline ADT & 666 & $-0,0729$ & $-0,0528$ & 0,2664 & 33 & $-0,0729$ & $-0,0528$ & 0,2664 & 0,0000 & 0,0000 \\
\hline CFO & 666 & 0,0601 & 0,0398 & 0,2662 & 33 & 0,0601 & 0,0398 & 0,2662 & 0,8038 & 0,1795 \\
\hline
\end{tabular}


CuADRo 3.-Control de los ajustes por devengo discrecionales (ADD) (cont.)

\begin{tabular}{|l|c|c|c|c|c|c|c|c|c|c|}
\hline ADD1 & 666 & 0,0244 & 0,0347 & 0,2511 & 33 & 0,0244 & 0,0347 & 0,2511 & 0,0001 & 0,0000 \\
\hline ADD2 & 666 & 0,0253 & 0,0376 & 0,2500 & 33 & 0,0253 & 0,0376 & 0,2500 & 0,0000 & 0,0000 \\
\hline ADD3 & 666 & 0,0289 & 0,0397 & 0,2511 & 33 & 0,0289 & 0,0397 & 0,2511 & 0,0001 & 0,0000 \\
\hline ADD4 & 666 & 0,0047 & 0,0217 & 0,2510 & 33 & 0,0047 & 0,0217 & 0,2510 & 0,0001 & 0,0000 \\
\hline ADD5 & 666 & 0,0048 & 0,0239 & 0,2505 & 33 & 0,0048 & 0,0239 & 0,2505 & 0,0001 & 0,0000 \\
\hline ADD6 & 666 & 0,0148 & 0,0320 & 0,2511 & 33 & 0,0148 & 0,0320 & 0,2511 & 0,0001 & 0,0000 \\
\hline
\end{tabular}

\begin{tabular}{|c|c|c|c|c|c|c|c|c|c|c|}
\hline \multicolumn{2}{|c|}{ Panel E: Empresas con pequeños beneficios versus empresas con elevados beneficios } \\
\hline & \multicolumn{3}{|c|}{ Pequeños beneficios $(0<\mathrm{RN}<0,03)$} & \multicolumn{3}{|c|}{ Extremo (RN>0,4) } & \multicolumn{3}{c|}{ Test-diferencias } \\
\hline Vbles & $\mathrm{N}$ & media & mediana & desv. & $\mathrm{N}$ & media & mediana & desv. & media & mediana \\
\hline ADT & 2.139 & $-0,0679$ & $-0,0578$ & 0,2358 & 106 & $-0,2478$ & $-0,1674$ & 0,6429 & 0,0049 & 0,0001 \\
\hline CFO & 2.139 & 0,0836 & 0,0726 & 0,2531 & 106 & 0,7240 & 0,6360 & 0,6224 & 0,0000 & 0,0000 \\
\hline ADD1 & 2.139 & 0,0218 & 0,0325 & 0,2258 & 106 & $-0,1101$ & $-0,0637$ & 0,6437 & 0,0378 & 0,0003 \\
\hline ADD2 & 2.139 & 0,0228 & 0,0346 & 0,2233 & 106 & $-0,1178$ & $-0,0661$ & 0,6258 & 0,0230 & 0,0001 \\
\hline ADD3 & 2.139 & 0,0244 & 0,0355 & 0,2258 & 106 & $-0,1268$ & $-0,0798$ & 0,6458 & 0,0180 & 0,0000 \\
\hline ADD4 & 2.139 & 0,0167 & 0,0223 & 0,2331 & 106 & $-0,1131$ & $-0,0507$ & 0,6382 & 0,0393 & 0,0017 \\
\hline ADD5 & 2.139 & 0,0174 & 0,0235 & 0,2307 & 106 & $-0,1174$ & $-0,0619$ & 0,6218 & 0,0282 & 0,0003 \\
\hline ADD6 & 2.139 & 0,0222 & 0,0281 & 0,2329 & 106 & $-0,1496$ & $-0,0958$ & 0,6424 & 0,0071 & 0,0000 \\
\hline
\end{tabular}

En el panel C (cuadro 3), se someten a contrastación los ajustes por devengo discrecionales de empresas con pequeñas pérdidas (medido por los cinco intervalos inmediatamente anteriores a cero) y empresas con pequeños beneficios (medidos por los cinco intervalos subsiguientes a cero $)^{6}$. Los resultados indican que no se puede aceptar la hipótesis de diferencias entre ambas muestras en todos los casos. Únicamente se alcanza la significatividad mínima requerida en dos modelos de ajustes por devengo a corto plazo. En este sentido, no puede defenderse categóricamente la idea de que los ajustes por devengo sean un instrumento para traspasar el umbral de rentabilidad de cero beneficios. Con otras palabras, si la obtención de pequeños beneficios es indicativo de una gestión del resultado, no todos los modelos de ajustes

${ }^{6}$ En las segmentaciones del cuadro 3 se han utilizado distintos intervalos para comprobar que los resultados se mantienen en la misma dirección. Así, se ha repetido el análisis utilizando los dos, tres y cuatro intervalos inmediatamente a la izquierda y a la derecha de cero. A medida que se disminuye la amplitud de los intervalos aumenta la insignificatividad de los ADD. 
por devengo son capaces de recoger dicha alteración o bien el punto de referencia fijado no es atribuible únicamente a una alteración del resultado (véase Coulton y otros 2005: 565-566).

Más aun, en el Panel D y E (cuadro 3) se examinan los ajustes discrecionales a la izquierda de la distribución (panel D) y a la derecha de la distribución (panel E). El panel D enfrenta empresas con pequeñas pérdidas (tres intervalos inmediatamente a la izquierda de cero) con empresas que declaran elevadas pérdidas (ratio superior a -0,4). Los resultados ponen de manifiesto diferencias entre ambas submuestras, en media y mediana $(p<0,01)$. Similar evidencia puede observarse a la derecha de la distribución (panel E) cuando se enfrentan empresas con pequeños beneficios y empresas con elevados beneficios.

En definitiva, parece que las empresas que justamente no alcanzan el punto de referencia (empresas con pequeñas pérdidas) y aquellas que justamente sobrepasan el punto de referencia (empresas con pequeños beneficios) no presentan el mismo nivel de ajustes por devengo (o alteración de resultados) cuando se les compara con el resto de empresas y con los extremos de las observaciones que pertenecen a su propio grupo. Sin embargo, no todos los modelos de ajustes por devengo son capaces de discriminar entre las empresas con pequeñas pérdidas y las empresas con pequeños beneficios. Esta evidencia sugiere que las medidas de ajustes por devengo deben tomarse con cierta precaución. Dicho con otras palabras, se debe ser cauto en la interpretación de la discontinuidad en las series de resultados como indicativo indiscutible de manipulación de resultados.

Por ultimo, los gráficos A-1 del anexo presentan un análisis gráfico de los ajustes por devengo que nos permite observar si éstos se encuentran relacionados con el crecimiento de la empresa (similares controles usan Dechow y otros 2003: 371, Coulton y otros 2005: 571). Para ello, se parte de la representación gráfica del resultado neto dividido por el activo total a principios del período con intervalos de 0,01. En el primer gráfico se representan los ajustes por devengo positivos para cada intervalo de resultado, a la izquierda y a la derecha de cero. En el segundo gráfico aparece la proporción de ajustes por devengo positivos a ambos lados de la distribución del resultado. El tercer gráfico parte de la representación anterior pero incluye los extremos de la distribución del resultado. En todos ellos, se observa que la magnitud o proporción los ajustes por devengo discrecionales positivos no depende de crecimiento de la empresa.

Cabe apuntar que trabajos previos tienen en cuenta variables de control que pueden afectar a las prácticas de gestión del resultado como el tamaño, el endeudamiento (Watts y Zimmerman 1986), y el crecimiento (Skinner y Sloan 2002) etc. Interesante también es el estudio de otro tipo de variables como puede ser la realización de proyec- 
tos de I+D, la edad, la reputación, la internacionalización, etc. El estudio de un conjunto de variables cualitativas y cuantitativas que motiven a los administradores a llevar a cabo prácticas de gestión del resultado escapa de los objetivos de este trabajo.

No obstante, se ha probado la relación que existe entre las prácticas de gestión del resultado y el endeudamiento empresarial. En los gráficos 5 se han representado las distribuciones de los resultados de la muestra en función del nivel de deuda que declaran al final del período contable. Para ello se ha dividido la muestra en empresas muy endeudadas y poco endeudadas. La partición se ha llevado a cabo usando los cuartiles ${ }^{7}$. El primer histograma de la izquierda, representativo de las empresas con menos endeudamiento (primer cuartil), refleja una discontinuidad en el punto cero pero no se presenta estadísticamente significativa. Lo contrario ocurre en el primer histograma de la derecha, representativo de las empresas con más endeudamiento (tercer cuartil), donde la discontinuidad es estadísticamente significativa. Similar evidencia se observa para cambios en resultados (véase los dos gráficos siguientes).

Los mismos pasos se han seguido en la representación del resultado pre-manipulación. Las dos últimas figuras de los gráficos 5 muestran una situación distinta a la anterior. En ambas se puede observar una distribución alisada en torno al punto de cero beneficios tanto para las empresas menos endeudadas como para las empresas más endeudadas. La discontinuidad no se confirma estadísticamente $(p>0.01)$. Además la comparación entre ambas distribuciones (resultados observados y pre-manipulacion) a través de la chi-cuadrado es estadisticamente significativa $(p<0.01)$. La evidencia obtenida apunta a que las empresas con mayor endeudamiento presentan una mayor predisposición a alterar los resultados con el objetivo de evitar pérdidas y descensos en resultados.

7 En un análisis adicional se han utilizado otras medidas como la media, mediana, etc. Los resultados obtenidos caminan en la misma dirección. 
GRÁfICO 5.--Incidencia del endeudamiento

Primer cuartil

Resultado neto $\left(\mathrm{RN}_{\mathrm{t}} / \mathrm{A}_{\mathrm{t}-1}\right)$

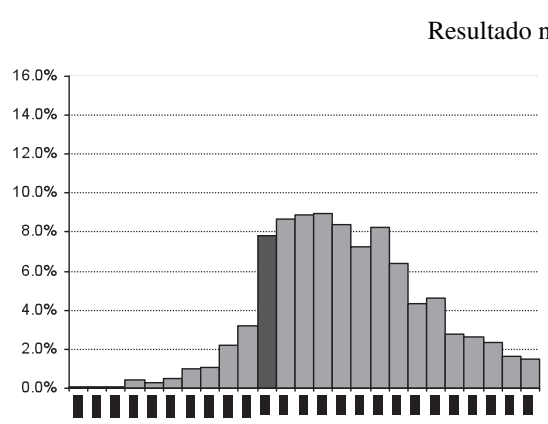

Tercer cuartil

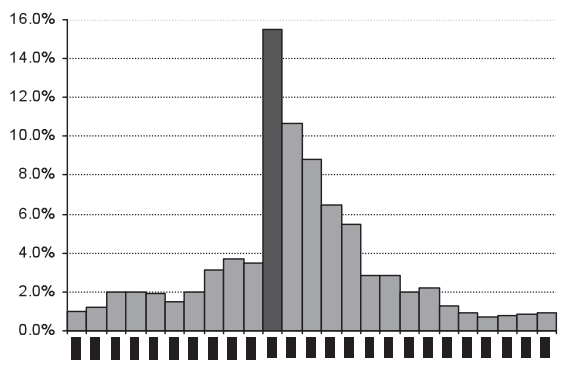

Cambios en Resultados netos $\left[\left(\mathrm{RN}_{\mathrm{t}}-\mathrm{RN}_{\mathrm{t}-1}\right) / \mathrm{A}_{\mathrm{t}-1}\right]$
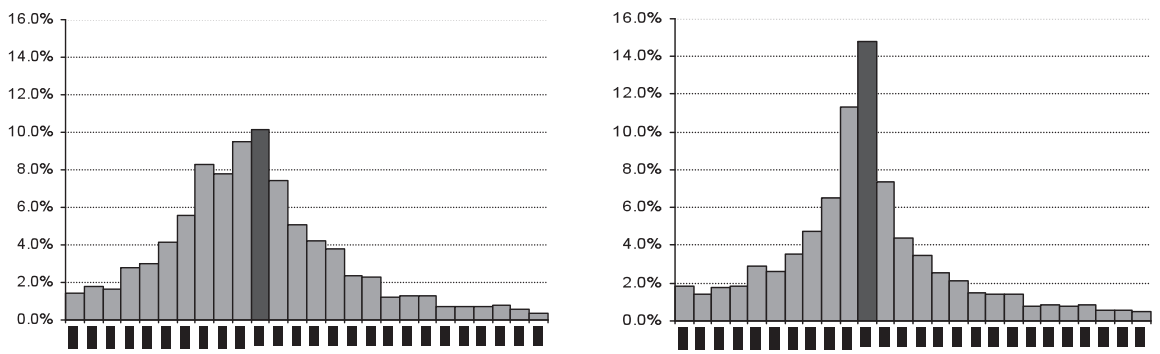

Resultados netos pre-manipulación $\left(\mathrm{RNp} 1_{\mathrm{t}} / \mathrm{A}_{\mathrm{t}-1}\right)$
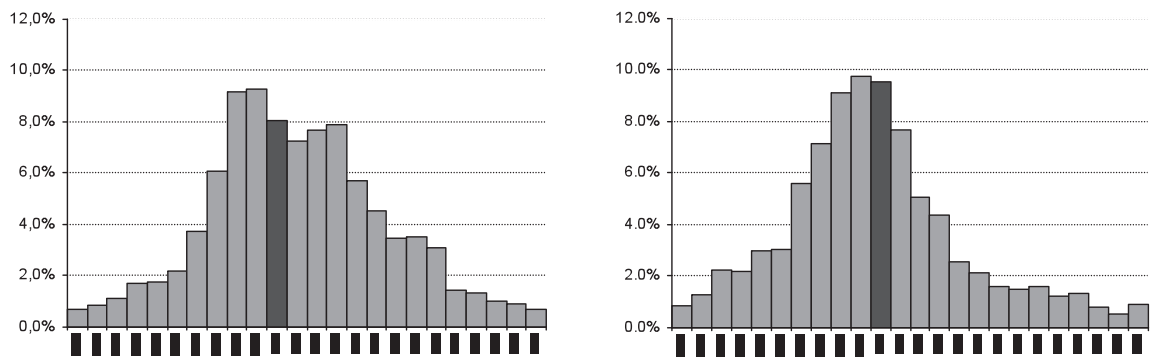

Nota: La amplitud de los intervalos se ha calculado de acuerdo con la fórmula de Degeorge y otros siendo de $(0,016)$ para el resultado neto $(\mathrm{RN})$ en el primer cuartil, de $(0,008)$ para el resultado neto en el tercer cuartil, de $(0.007)$ para cambios en resultados netos $(\Delta \mathrm{RN})$ en el primer y tercer cuartil; de $(0,037)$ para el resultado pre-manipulación $(\mathrm{RNp} 1)$ en el primer cuartil y de $(0,034)$ para el resultado pre-manipuación en el tercer cuartil. Para la definición de los resultados pre-manipulación $(\mathrm{RNp})$ véase la nota a pie de página de los Gráficos 3. 


\subsection{Análisis de sensibilidad: Control de los histogramas de frecuencias}

\subsubsection{Control sobre la amplitud de los intervalos}

Los estudios iniciales atribuían la existencia de una discontinuidad en torno a cero resultados a una gestión por parte de los administradores en aras a declarar pequeños beneficios y pequeños incrementos en resultados frente a pequeñas pérdidas y pequeños descensos en resultados. Sin embargo, algunas publicaciones fechadas a mediados de la década del 2000 dan cuenta de una serie de problemas asociados a los histogramas de frecuencias y en este contexto, se hace necesario llevar a cabo una serie de controles sobre la amplitud de los intervalos y el denominador de los resultados.

Holland (2004) considera que la elección de la amplitud del intervalo en la representación gráfica de los histogramas es un punto crítico en la investigación de la gestión del resultado en los puntos tomados como referencia por lo que conviene emplear distintas alternativas. Este autor propone usar cinco métodos para determinar la amplitud de los intervalos bajo el supuesto de una distribución normal: 3,5 $\mathrm{\sigma n}^{-1 / 3}$ (Scott $1979), 2(\mathrm{IQR}) \mathrm{n}^{-1 / 3}$ (Freedman-Diaconis 1981), ${ }_{-1 / 5}, 06 \mathrm{n}^{-1 / 5}$ (Silverman 1986), 0,79(IQR) $\mathrm{n}^{-1 / 5}($ Silverman 1986), y 0,9(min $(\sigma \mathrm{IQR} / 1.34)) \mathrm{n}^{-1 / 5}($ Silverman 1986). Donde $\sigma=$ desviación estándar de la muestra, $\mathrm{IQR}=$ rango inter-cuartílico de la muestra y $\mathrm{n}=$ número de observaciones ${ }^{8}$.

En este trabajo, se ha utilizado la fórmula de Degeorge y otros (1999) para determinar la amplitud de los intervalos y posteriormente se ha comprobado que la evidencia se mantiene sin grandes variaciones utilizando distintas alternativas $(0,01,0,05,0,005$, así como fijar de antemano un número constante de barras en todos los histogramas y permitir que la amplitud del intervalo fluctué en función de ellas) ${ }^{9}$. Los gráficos A-2 del anexo muestran las distribuciones de niveles y cambios de resultados divididos por el activo total a principio de año usando intervalos de 0,01 para niveles y 0,005 para cam-

${ }^{8}$ En un análisis de sensibilidad se han comprobado las medidas propuestas por Silverman (1986) y Scott (1992). Estos autores señalan varias formas para calcular la amplitud de los intervalos relacionadas positivamente con la variabilidad de los datos y negativamente con el número de observaciones.

9 Veáse las indicaciones de Burgstahler y Dichev (1997) y las recomendaciones de Holland (2004) para el cálculo de la amplitud de los intervalos. 
bios de resultados. La evidencia encontrada sigue mostrando una discontinuidad alrededor de cero beneficios y significativa estadísticamente lo que respalda los resultados previos. De nuevo, en la representación gráfica de las series de descensos en resultados la discontinuidad en torno a cero cambios no es tan evidente. A este respecto todavía no sería oportuno emitir opinión alguna sobre la hipótesis de evitar pérdidas y descensos en resultados puesto que no acaban aquí los problemas asociados a la metodología de los histogramas de frecuencias. En el siguiente apartado se examina una de las debilidades más estudiadas en el momento presente: el denominador de los resultados.

\subsubsection{Control sobre el denominador de los resultados}

Estudios recientes consideran que la discontinuidad observada en los puntos críticos en la representación gráfica de las funciones de densidad de los resultados puede que no se deba a una alteración del resultado por parte de los administradores. Por ejemplo, Degeorge y otros (1999) sugieren que el denominador utilizado para dividir los resultados provoca una dispersión en las observaciones que puede contribuir a generar artificialmente dicha discontinuidad en el punto cero. Durtschi y Easton (2005) utilizan el valor de mercado para transformar los resultados brutos en un ratio de resultados comparable empresa a empresa y consideran que dicho denominador puede ser el responsable de la discontinuidad observada en el punto cero beneficios. Estos autores encuentran que las empresas con pérdidas son valoradas de distinta manera que las empresas con beneficios. Dicho con otras palabras, las empresas situadas a la izquierda del punto cero tienen distinto denominador (valor de mercado, activo o ventas) que las observaciones situadas a la derecha del punto cero, generando una discontinuidad en los histogramas. Incluso si la distribución de los resultados no mostrara una discontinuidad, el efecto de transformar la variable bruta resultados en una variable relativa (mediante el valor de mercado, el activo o las ventas) provocaría su aparición.

Siguiendo las indicaciones de estos autores se han dividido los tres tipos de resultados usados en este trabajo por las ventas al principio y al final del período contable y por el activo al final del período. En los cuatro primeros histogramas de los gráficos A-3 del anexo se observa una discontinuidad en el punto de cero beneficios (y cero cambios en beneficios), significativa estadísticamente $(p<0,05)$.

En el cuadro A-1 del anexo se toma como base la representación del resultado neto dividido entre el activo total a principios de año (véanse los gráficos 1) y se lleva a cabo un control del denominador (el activo en este caso) mediante una serie de segmentacio- 
nes a ambos lados del histograma ${ }^{10}$. En el panel A (cuadro A-1), se compara el activo y las ventas de las empresas que reportan pérdidas $(\mathrm{RN}<0)$ frente a aquellas que reportan beneficios $(\mathrm{RN}>0)$. En contra de lo esperado, se observa que las empresas rentables no presentan una inversión más elevada que las empresas no rentables. El panel A muestra que las empresas con pérdidas tienen un activo de 15.145 y 2.652 miles de euros en media y mediana respectivamente, y las empresas con pérdidas tienen un activo de 8.118 y 2.278 miles de euros en media y mediana, respectivamente ${ }^{11}$. Sin embargo, las diferencias en media y mediana se presentan significativas $(p<0,01)$. De otro lado, las ventas en empresas no rentables alcanzan los valores de 4.092 y 1.425 miles de euros, en media y mediana respectivamente mientras que en las empresas rentables alcanzan los valores de 3.904 y 1.648 miles de euros respectivamente. Las diferencias en media y mediana no se muestran significativas $(p>0,01)$.

En los paneles B y C (cuadro A-1) se compara, en términos de activo y ventas, los cinco intervalos inmediatamente a la izquierda (pequeñas pérdidas) y a la derecha de cero (pequeños beneficios). Los datos encontrados ponen de manifiesto que las primeras no presentan una inversión (ventas) significativamente mayor que las segundas, tanto en media como en mediana $(p>0,01)$. En los paneles D y E (cuadro A-1) se contrastan los extremos de la distribución. Esto es, el activo y las ventas de las empresas con mayores beneficios (tres últimos intervalos) en relación al de las empresas con mayores pérdidas (tres últimos intervalos). De nuevo, se observa que no existen diferencias en media y mediana entre ambas submuestras $(p>0,01)$

También se ha comprobado el efecto del tamaño (inversión y ventas) a la izquierda y a la derecha de la distribución del punto cero (paneles F y G, cuadro A-1). En la vertiente izquierda se han enfrentado empresas con elevadas pérdidas (ratio superior a $-0,4$ ) y empresas con pequeñas pérdidas (ratio entre 0,00 y $-0,033$ ) y no se encuentran

10 Similar control se ha efectuado tomando como punto de partida la representación del resultado neto dividido por las ventas a principios del año. Los resultados caminan en la misma dirección.

${ }^{11}$ El sector hotelero español se caracteriza por su atomización debido a la escasa dimensión de buena parte de las empresas dedicadas a actividades turísticas, pero asistimos a un escenario en el que algunas compañías españolas, especialmente cadenas hoteleras, se enfrentan a procesos importantes de internacionalización de su actividad que necesariamente exigen una reconsideración de sus estrategias económicas y financieras. Así, Sol Melia se encuentra clasificada en la zona de pérdidas en los períodos 2001, 2002 y 2003 provocando, junto con otras cadenas hoteleras, que la media y mediana de la zona de pérdidas supere a la de la zona de beneficios a la vez que una elevada desviación típica. 
diferencias en media y mediana $(p>0,01)$. Del mismo modo, en la vertiente derecha se han enfrentado empresas con elevados beneficios (ratio superior a 0,4 ) y empresas con pequeños beneficios (ratio entre 0,00 y 0,033 ) y no se observan diferencias en media y mediana $(p>0,01)$.

Entonces, en contra de las predicciones de Durtschi y Easton's (2005), el denominador de los resultados (el activo al inicio del año) en el sector hotelero español no muestra diferencias estadísticamente significativas a ambos lados del punto crítico lo que generaría automáticamente una discontinuidad al mover observaciones de la zona de pequeñas pérdidas hacia el punto cero y alejando de este punto las observaciones situadas en la zona de pequeños beneficios (en el mismo sentido, Kerstein y Rai 2007).

Por último, se ha representado gráficamente la distribución del resultado en valores brutos (último histograma de los gráficos A-3) con intervalos de 24,94 resultante de aplicar la fórmula propuesta por Degeorge y otros (1999). En contra de lo esperado, se sigue manteniendo la discontinuidad en torno a cero resultados y alcanza los niveles estadísticos requeridos $(\mathrm{p}<0,01)$. Además, no se puede aceptar la hipótesis de igualdad de muestras en las regiones de pérdidas y beneficios, realizada mediante la prueba de la chi-cuadrado, puesto que se alcanza un nivel de significatividad mayor del 1 por ciento $(p=0,0000)$. Lo mismo sucede si se comprueba en las zonas críticas de pequeñas pérdidas frente a pequeños beneficios. Por tanto, la distribución del resultado en la vertiente izquierda del punto cero es distinta a la distribución del resultado en la vertiente derecha del cero.

En suma la evidencia obtenida en este apartado sugiere que el denominador de los resultados no provoca la irregularidad en torno a cero resultados en la muestra manejada en este estudio. Cabe mencionar que los resultados obtenidos solamente son extensibles al sector hotelero español y la elección de otro tipo de muestras probablemente dé lugar a evidencias en otra dirección (véase Holland 2004, Durtschi y Easton 2005 y Hann y Lu 2007, entre otros).

\section{CONCLUSIONES}

El objetivo de este trabajo es comprobar la gestión del resultado en torno a evitar pérdidas y descensos en resultados en una muestra de empresas hoteleras españolas. Desde los trabajos seminales de Hayn (1995) y Burgstahler y Dichev (1997), se ha demostrado satisfactoriamente la gestión del resultado en estos umbrales de rentabilidad que se consideran críticos en un amplio conjunto de países con muestras sujetas a distintas restricciones. Cabe apuntar que la mayor parte de las evidencias se han centrado 
en Estados Unidos y en sectores donde existe relativa facilidad de contar con información de tipo financiero y no financiero como es el caso de las empresas cotizadas.

No obstante, todavía quedan muchos sectores de la economía sin explorar y la evidencia de las prácticas de gestión por parte de los administradores es escasa o incluso nula. La contribución del sector turístico en la economía española no se puede tildar de escasa o insignificante siendo muchos los autores que consideran que es la principal actividad económica española. Cualquier aproximación empírica dentro de las empresas que conforman el sector turístico en general u hotelero en particular puede resultar de gran utilidad para un mejor conocimiento de la industria, a pesar de las dificultades que presenta la recogida de la información y su fiabilidad.

En primer lugar, en este trabajo se han representado las distribuciones de niveles y cambios de resultados para comprobar la existencia de una discontinuidad en torno a cero beneficios (evitar declarar pérdidas) y cero cambios (evitar declarar descensos en resultados). Los histogramas construidos para tres niveles de resultados (ordinario, antes de impuestos y neto) de la cuenta de pérdidas y ganancias divididos por el activo total a principios de año, ponen de manifiesto que existe un punto de discontinuidad en cero beneficios, estadísticamente significativo. La discontinuidad en el punto de cero cambios en resultados es menos evidente que en el caso de niveles.

En segundo lugar, se han estimado tres modelos de ajustes por devengo, Jones original (1991), Jones modificado (Dechow y otros 1995) y el modelo de Kothari y otros (2005), tanto a largo como a corto plazo, lo que nos permite obtener los resultados premanipulación y compararlos con los resultados declarados. La representación gráfica de los resultados pre-manipulación muestra una distribución más o menos alisada en torno a cero beneficios muy distinta a la encontrada en la representación de los resultados declarados en las cuentas anuales.

El análisis de los ajustes por devengo discrecionales indica que las empresas que justamente sobrepasan (no alcanzan) el umbral de cero beneficios presentan ajustes discrecionales positivos y más elevados que el resto de empresas y frente a aquellas de su mismo grupo que se sitúan en los extremos del histograma. No obstante, cuando se comparan los ajustes por devengo discrecionales de las empresas que justamente sobrepasan el umbral de cero beneficios (zona de pequeños beneficios) frente a las que justamente no lo alcanzan (zona de pequeñas pérdidas) no se muestran significativos en todos los casos. Quizás la estimación de modelos de ajustes por devengo que se ajusten en mayor medida a las especificidades del sector o la inclusión de nuevas variables distintas a los ajustes por devengo arrojen una evidencia más clara sobre esta cuestión. La representación gráfica de los ajustes discrecionales (utilizando cualquiera de las especificaciones propuestas) muestra que éstos son mayores en las empresas que satis- 
factoriamente sobrepasan el punto crítico y además dicha representación gráfica pone de manifiesto que no que existe una relación directa con el crecimiento de la empresa tal y como demuestran Dechow y otros (2003) y Coulton y otros (2005).

Por último, al hilo de las publicaciones de autores de la talla de Dechow y otros (2003), Holland (2004), Durtschi y Easton (2005) y Beaver y otros (2007), entre otros, se ha llevado a cabo una serie de controles sobre la evidencia preliminar obtenida de la representación gráfica de las distribuciones de resultados que permiten robustecer los resultados. Estos autores ponen en entredicho que la discontinuidad observada en las series de resultados derive de una gestión intencionada por parte de los administradores. A pesar de que dicha discontinuidad podría ser consistente con la gestión del resultado para evitar pérdidas y descensos en resultados no se considera suficiente para afirmar rotundamente que existe una alteración de resultados.

En este sentido es necesario establecer una serie de controles en el denominador de los resultados, en los intervalos utilizados en la representación de los resultados, etc. La utilización de distintos intervalos de resultados y el uso de diferentes variables para dividir los resultados no alteran la evidencia previa. Incluso cuando se representan los resultados brutos se sigue manteniendo la discontinuidad en torno a cero beneficios y se observa una asimetría en la distribución del resultado. Si bien cabe comentar que los denominadores de los resultados no se muestran exentos de mover observaciones de una zona a otra en la distribución de los resultados por lo que estudios posteriores deben tener en cuenta su efecto.

La utilización de las dos metodologías líderes en la detección de la alteración del resultado - los histogramas de frecuencias junto con tres modelos de ajustes por devengo - apunta a que los gerentes no se muestran indiferentes ante la cifra (variación) de resultados publicada. Una amplia literatura puede verse en relación con las elevadas motivaciones que justifican ciertos retoques en la variable contable que a lo largo del tiempo ha tenido el privilegio de influir en las decisiones de un amplio conjunto de usuarios financieros. En la hotelería española los resultados encontrados pueden venir justificados por el acceso a fuentes de financiación dentro del proceso de expansión en el que se encuentra inmerso el sector y los escasos controles que existen sobre la información financiera en un sector caracterizado por la atomización de las empresas que lo conforman.

Estudios de corte internacional ponen de manifiesto que los países clasificados dentro del modelo continental tienen más predisposición a alterar el resultado que los países anglosajones (Leuz y otros 2003). En este sentido, se piensa que cualquier aproximación empírica que permita acercarnos al conocimiento de la realidad empresarial queda justificada más aun teniendo en cuenta que el estudio de las prácticas de gestión 
del resultado todavía se encuentra en una fase incipiente en buena parte de los sectores que conforman el tejido empresarial en nuestro país. Futuros trabajos pueden comprobar qué tipo de variables — financieras y de otra índole — tienen mayor propensión a ser utilizadas por los gerentes para alcanzar con efectividad sus propósitos. Interesante también sería llevar a cabo un análisis comparativo tomando como base una muestra representativa del sector hotelero de un conjunto de países. Asimismo, es necesario profundizar en las técnicas de detección de las prácticas de gestión del resultado ya que como indican los últimos trabajos publicados, pueden dar lugar a sesgos a favor de la gestión del resultado.

\section{REFERENCIAS BIBLIOGRÁFICAS}

Ayers, B.; Jiang, J. y Yeung, E. (2006): «Discretionary accruals and Earnings Management: an analysis of pseudo earnings target», The Accounting Review, vol. 81 (3): 617-653.

Beatty, A.; Ke, B. y Petroni, K. (2002): «Earnings Management to avoid earnings declines across publicly and privately held banks», The Accounting Review, vol. 77 (3): 547-570.

Beaver, W. H.; Mcnichols, M. F. y Nelson, K.K (2003): «Management of the loss reserve accrual and the distribution of earnings in the property-casualty insurance industry», Journal of Accounting and Economics, vol. 35 (3): 347-376.

- (2007): «An alternative interpretation of the discontinuity in earnings distributions», Review of Accounting Studies, vol. 12 (4): 525-556.

Burgstahler, D. y Dichev, I. (1997): «Earnings Management to avoid earnings decreases and losses», Journal of Accounting and Economics, vol. 24 (1): 99-126.

CoAse, R. H. (1937): «The nature of the firm», Economica, vol. 4 (16): 386-405.

Coppens, L. y Peek, E. (2005): «An analysis of Earnings Management by European Private Firms», Journal of International Accounting, Auditing and Taxation, vol. 14 (1): 1-17.

Coulton, J.; TAYlor, S. y TAYlor, S. (2005): «Is Benchmark Beating by Australian Firms Evidence of Earnings Management?», Accounting and Finance, vol. 45 (4): 553-576.

Dechow, P.; Sloan, R. G. y Sweeney, A. P. (1995): «Detecting Earnings Management», The Accounting Review, vol. 70 (2): 193-225.

Dechow, P. M.; Richardson, S. A. y Tuna, I. A. (2003): «Why are Earnings Kinky? An Examination of the Earnings Management Explanation», Review of Accounting Studies, vol. 8 (2-3): 355-384.

Degeorge, F.; Patel, J. y Zeckhauser, R. (1999): «Earnings Management to exceed thresholds», Journal of Business, vol. 72 (1): 1-33. 
Durtschi, C. y Easton, P. (2005): «Earnings Management? The Shapes of the Frequency Distributions of Earnings Metrics are not Evidence Ipso Facto», Journal of Accounting Research, vol. 43 (4): 557-592.

FIELDS, T.; Lys, L. y VICENT, L. (2001): «Empirical research on accounting choice», Journal of Accounting and Economics, vol. 31 (1-3): 255-307.

Gallén, M. L. y Giner, B. (2005): «La alteración del resultado a través del análisis de la distribución de frecuencias», Revista Española de Financiación y Contabilidad, vol. 124, EneroMarzo: 141-181.

Gill De Albornoz, B. e Illueca, M. (2003): «Regulación de precios y prácticas de Earnings Management: evidencia empírica en el sector eléctrico español», Revista Estudios Financieros, vol. 247 (62), Octubre: 155-192.

- (2007): «La Calidad de los ajustes por devengo no afecta al coste de la deuda de las Pymes españolas», Investigaciones Económicas, vol. XXXI (1): 79-117

Gore, P.; Pope, P. y Singh, A. (2007): «Earnings Management and the Distribution of Earnings relative to targets», Accounting and Business Research, vol. 37 (2): 123-150.

HAYN, C. (1995): «The Information content of losses», Journal of Accounting and Economics, vol. 20 (2): 125-153.

Hann, R. N. y Lu, Y. Y. (2007): «Earnings Management at the Segment Level», University of Southern California.

Healy, P. y Wahlen, J. M. (1999): «A review of the Earnings Management literature and its implications for standard setting», Accounting Horizons, vol. 13 (4): 365-383.

Holland, D. y Ramsay, A. (2003): «Do Australian companies manage earnings to meet simple Earnings Benchmarks», Accounting and Finance, vol. 43 (1): 41-62.

Holland, D. (2004): «Earnings Management: a methodological review of the distribution of reported earnings approach», Working Paper, Monash University of Australia.

JACOB, J. y JoRgEnSEN, B. N. (2007): «Earnings Management and accounting income aggregation», Journal of Accounting and Economics, vol. 43 (2-3): 369-390.

JoNES, J. J. (1991): «Earnings Management during import relief investigations», Journal of Accounting Research, vol. 29 (2): 193-228.

Kahneman, D. y Tversky, A. (1979): «Prospect Theory: an analysis of decision under risk», Econometrica, vol. 47 (2): 263-291.

Kahneman, D.; Knetsch, J. y Thaler, R. (1991): «The endowment effects, loss aversion, and status quo bias», Journal of Economic Perspectives, vol. 5 (1):193-206.

Kerstein, J. y RAI, A. (2007): «Intra-Year Shifts in the Earnings Distribution and their implications for Earnings Management», Journal of Accounting Economics, vol. 44 (3): 399-419. 
Kinnunen, J. y Koskela, M. (2003): «Who is Miss World in Cosmetic Earnings Management? A Cross-National comparison of small upward rounding of net income Numbers among eighteen Countries», Journal of International Accounting Research, vol. 2 (1): 39-68.

Kothari, S. P.; Leone, A. y Wasley, C. (2005): «Performance Matched Discretionary Accruals», Journal of Accounting Economics, vol. 39 (1): 163-197.

Leone, A. J. y Van Horn, R. L. (2005): «How Do Non-Profit Hospitals Manage Earnings?», Journal of Health Economics, vol. 24 (4): 815-837.

Leuz, C.; NAnda D. y Wysocki, P. (2003): «Earnings Management an Investor Protection: An International Comparison», Journal of Financial Economics, vol. 69 (3): 505-527.

LEv, B. (1989): «On the Usefulness of Earnings: Lessons and Directions from two decades of Empirical Research», Journal of Accounting Research, vol. 27 (Supplement): 153-192.

Mcnichols, M. y Wilson, P. (1988): «Evidence of Earnings Management from the provision for bad debts», Journal of Accounting Research, vol. 26, Supplement: 1-31.

Marquardt, C. A. y Wiedman, C. I. (2004): «How are Earnings Managed? An Examination of Specific Accruals», Contemporary Accounting Research, vol. 21 (2): 461-491.

Parte, L.; Gonzalo, M. C. y Gonzalo, J. A. (2007): «La hipótesis de la utilización del impuesto sobre beneficios para evitar pérdidas y descensos en resultados», Revista de ContabilidadSpanish Accounting Review, vol. 10 (2): 33-74.

PARTE, L. (2008): «The hypothesis of avoiding losses and decreases in earnings via extraordinary items», Revista Española de Financiación y Contabilidad-Spanish Journal of Finance and Accounting, vol. 139: 405-431.

Thomas, W. B.; Herrmann, D. R. y Inoue, T. (2004): «Earnings Management through affiliated transactions», Journal of International Accounting Research, vol. 3 (2): 1-25.

Tversky, A. y Kanhneman, D. (1991): «Loss Aversion in Riskless Choice: A Reference-Dependent Model», The Quarterly Journal of Economic, vol. 106 (4): 1039-1061.

Scotт, D. W. (1992): Multivariate Density Estimation: Theory, Practice, and Visualization, Wiley, New York.

SKINNER, D. J. y SLOAN, R., «Earnings surprises, growth expectations, and stock returns or don't let an earnings torpedo sink your portfolio», Review of Accounting Studies, vol. 7 (2-3): 289$312,2002$.

Silverman, B. W. (1986): Density estimation for statistics and data analysis, Chapman \& Hall, London.

Suda, K. y Shuto, A. (2005): «Earnings management to avoid earnings decreases and losses: empirical evidence from Japan», Rcss Discussion Paper Series.

Watts, R. y Zimmerman, J. (1986): Positive Accounting Theory, Prentice Hall, New York. 


\section{APÉNDICE}

GráfICo A-1.—Control de los ajustes por devengo discrecionales (ADD)
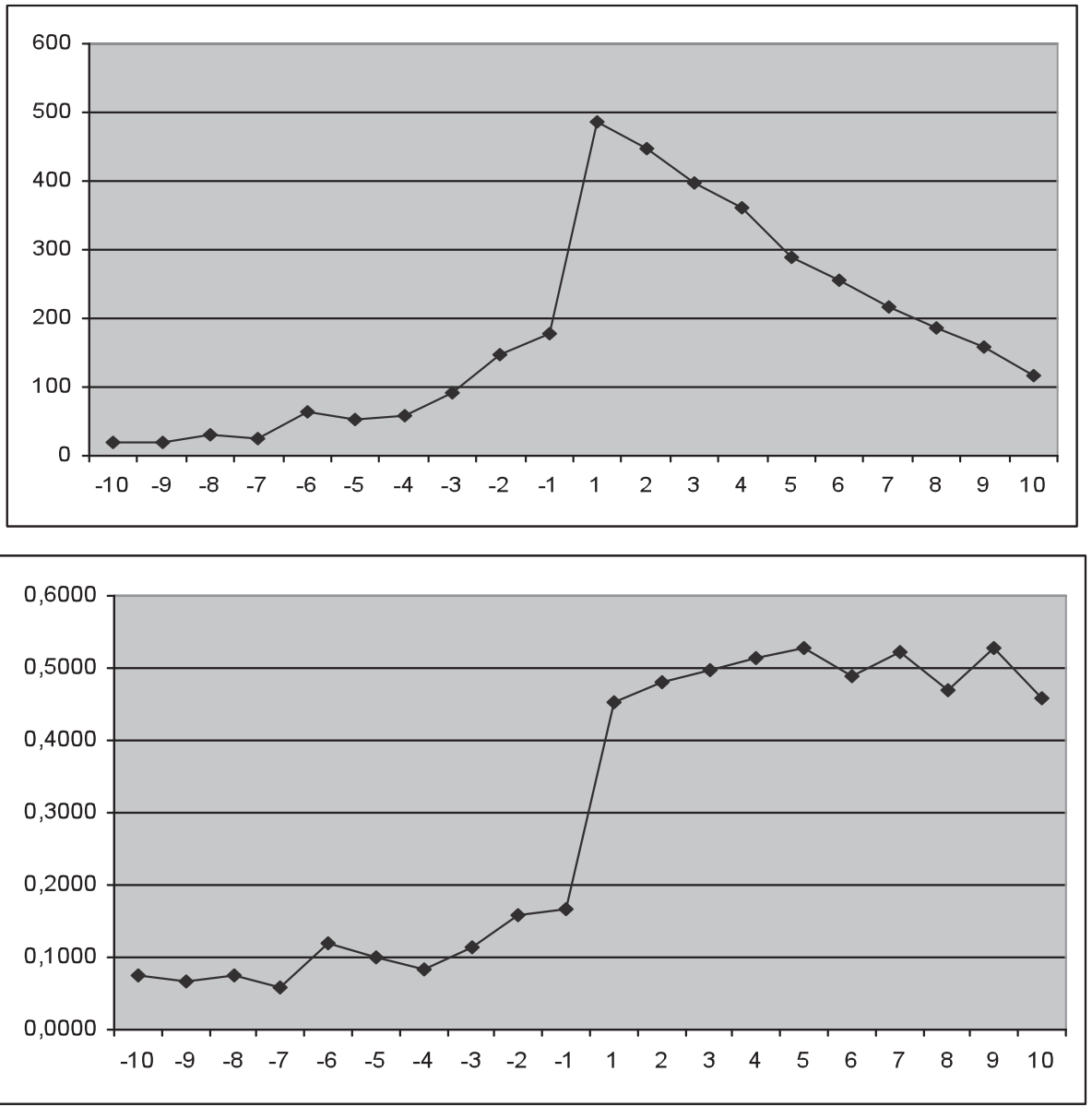
GráfICo A-1.—Control de los ajustes por devengo discrecionales (ADD) (cont.)

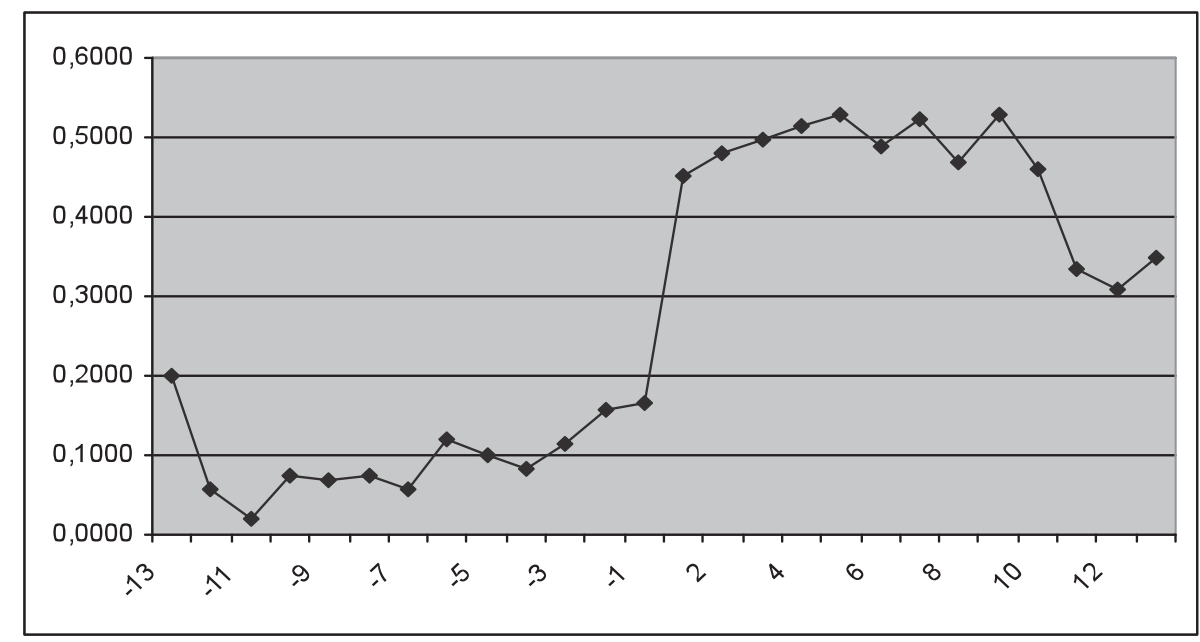

El primer gráfico toma como base la distribución del resultado neto dividido por el activo total y muestra los ajustes por devengo discrecionales positivos a ambos lados del punto cero beneficio. El segundo gráfico plantea la proporción de los ajustes por devengo discrecionales positivos en cada intervalo de resultado. El tercer gráfico, por su parte, permite ver la proporción de los ajustes por devengo discrecionales positivos en cada nivel de resultados donde se han incluido los extremos del histograma. Los intervalos son de 0,01 . Los ajustes por devengo discrecionales han sido calculados utilizando el modelo de Jones original (1991). Los mismos resultados se obtienen con los otros modelos de ajustes por devengo estimados. 
GrÁfICO A-2.-Análisis del efecto de la amplitud de los intervalos
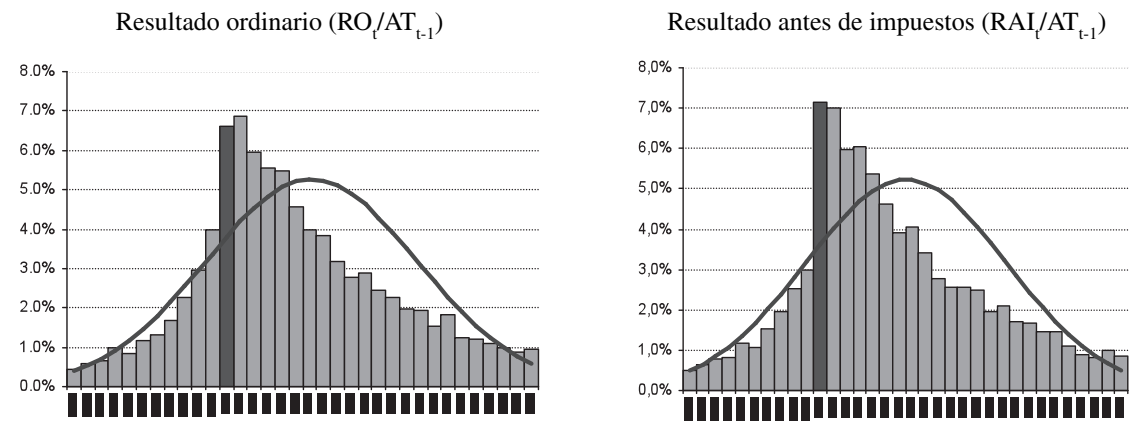

Resultado neto $\left(\mathrm{RN}_{\mathrm{t}} / \mathrm{AT}_{\mathrm{t}-1}\right)$

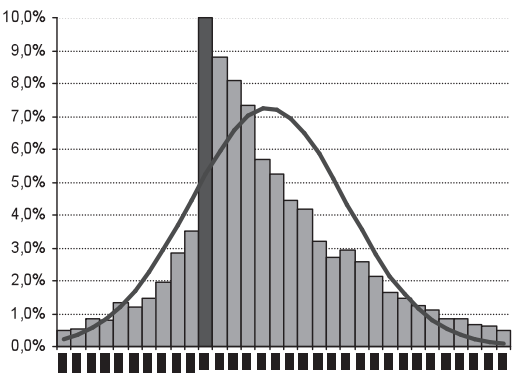

Cambios en resultados ordinarios $\left[\left(\mathrm{RO}_{\mathrm{t}}-\mathrm{RO}_{\mathrm{t}-1}\right) / \mathrm{AT}_{\mathrm{t}-1}\right]$ Cambios en resultados netos $\left[\left(\mathrm{RN}_{\mathrm{t}}-\mathrm{RN}_{\mathrm{t}-1}\right) / \mathrm{AT}_{\mathrm{t}-1}\right]$
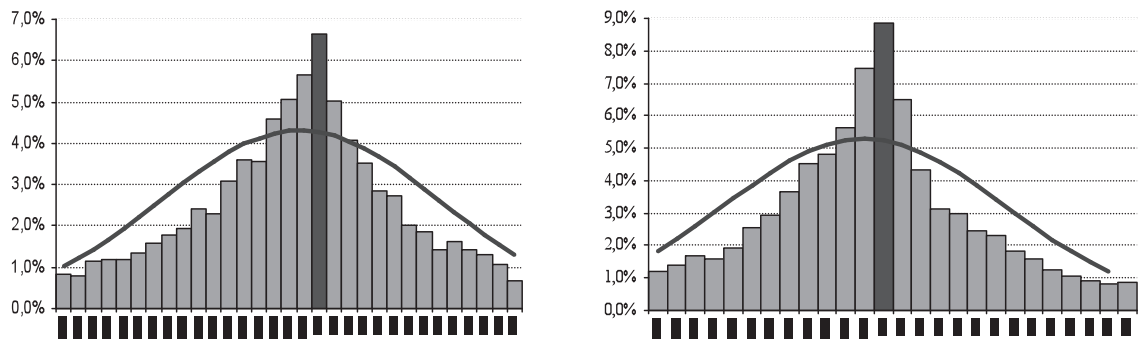

Nota: Los histogramas se han elaborado con datos pool a partir de 9.950 obs. La amplitud de los intervalos de los histogramas toman el valor de 0,01 para niveles de resultados y de 0,005 para cambios en resultados. Las diferencias estandarizadas siguiendo las indicaciones de Burgstahler y Dichev (primer número) y Beaver y otros (segundo número) son de: RO $(3,54$ y 3,16), RAI $(6,38$ y 5,75), RN $(9,96$ y 9,00), $\Delta \mathrm{RO}$ (4.03 y 3.61), $\Delta \mathrm{RN}$ (5.00 y 4.51). La línea continua representa la distribución obtenida, sobre el supuesto de normalidad, dada la media de la población y la desviación típica de una muestra que concentra el $90 \%$ de los valores en torno a esta media, evitando así el efecto que sobre la dispersión tendrían los valores extremos. Todos los histogramas presentan asimetría y curtosis positivas. Las colas están truncadas. La definición de las variables se encuentra en la nota del cuadro 1. 
GRÁFICo A-3.-Efecto del denominador de los resultados

$\mathrm{RN} / \mathrm{Vtas}_{\mathrm{t}}$

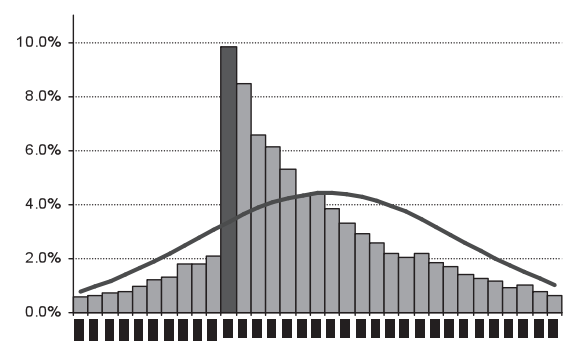

$\mathrm{RN}_{\mathrm{AT}}$

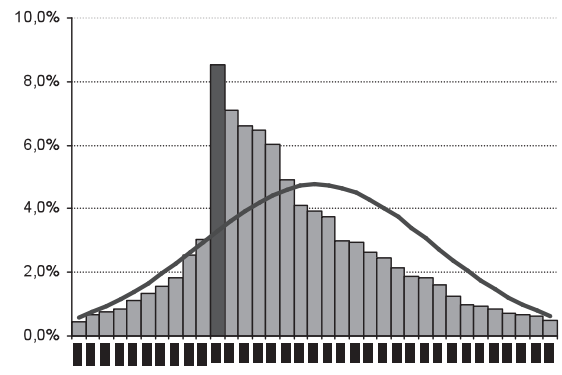

$\mathrm{RN} / \mathrm{Vtas}_{\mathrm{t}-1}$

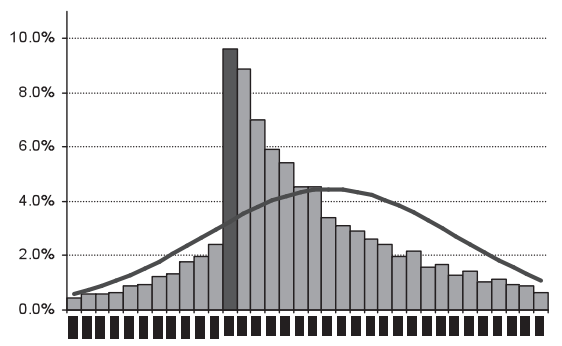

$\left[\left(\mathrm{RN}_{\mathrm{t}}-\mathrm{RN}_{\mathrm{t}-1}\right) / \mathrm{Vtas}_{\mathrm{t}-1}\right]$

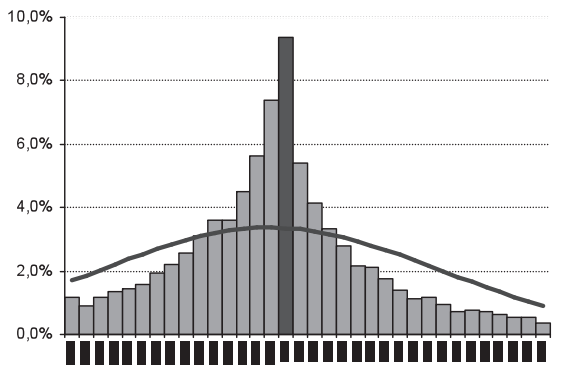

Resultado neto (en valores brutos)

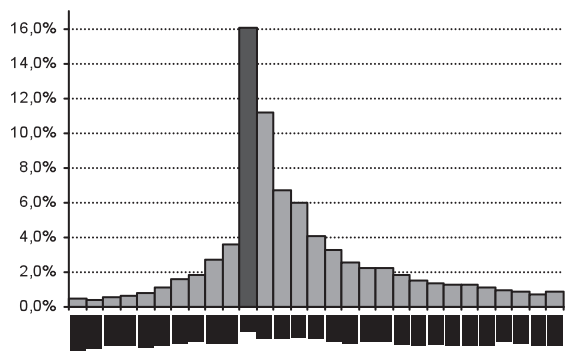

Nota: Los histogramas se han elaborado con datos pool a partir de 9.950 obs. Para el cálculo de la amplitud de los intervalos se ha seguido el criterio de Degeorge y otros (1999). La línea continua representa la distribución obtenida, sobre el supuesto de normalidad, dada la media de la población y la desviación típica de una muestra que concentra el $90 \%$ de los valores en torno a esta media, evitando así el efecto que sobre la dispersión tendrían los valores extremos. Las amplitudes de los intervalos toman valores de: $(0,010)$ para el resultado neto dividido por las ventas del año; $(0,011)$; para las ventas del año t-1; $(0,076)$ para el resultado neto dividido por el activo del año; $(0,096)$ para el cambio del resultado neto dividido por las ventas del año t-1 y 24,94 para el resultado neto en valores brutos. 
Las diferencias estandarizadas siguiendo las indicaciones de Beaver y otros (2007) toman los valores de: 11,08 (RN/Vtas $), 9,53\left(\mathrm{RN} / \mathrm{Vtas}_{\mathrm{t}-1}\right), 8,78\left(\mathrm{RN} / \mathrm{AT}_{\mathrm{t}}\right), 10,06\left(\Delta \mathrm{RN} / \mathrm{Vtas}_{\mathrm{t}}\right), 17,48(\mathrm{RN}$ bruto). Todos los histogramas presentan asimetría y curtosis positivas. Las colas están truncadas. Los mismos resultados se obtienen si se utilizan intervalos con distinta amplitud y si se representan las series de resultados ordinarios o antes de impuestos.

Cuadro A-1.—Análisis del denominador de los resultados: Activo $\left(\mathrm{AT}_{\mathrm{t}-1}\right)$

\begin{tabular}{|c|c|c|c|c|c|c|c|c|c|c|c|}
\hline \multicolumn{12}{|c|}{ Panel A: Empresas con pérdidas versus empresas con beneficios } \\
\hline RN & \multicolumn{4}{|c|}{ Empresas con pérdidas } & RN & \multicolumn{4}{|c|}{ Empresas con beneficios } & \multicolumn{2}{|c|}{ Test-diferencias } \\
\hline Intervalo & $\mathrm{N}$ & media & mediana & desv & Intervalo & $\mathrm{N}$ & media & mediana & desv & media & mediana \\
\hline Activo $(\mathrm{RN}<0)$ & 1.462 & 15.145 & 2.652 & 102.343 & Activo (RN>0) & 6.498 & 8.118 & 2.278 & 35.037 & 0,0096 & 0,0000 \\
\hline Ventas $(\mathrm{RN}<0)$ & 1.390 & 4.092 & 1.425 & 23.896 & Ventas $(\mathrm{RN}>0)$ & 6.474 & 3.904 & 1.648 & 12.838 & 0,6769 & 0,4747 \\
\hline
\end{tabular}

\begin{tabular}{|c|c|c|c|c|c|c|c|c|c|c|c|}
\hline \multicolumn{7}{|c|}{ Panel B: Empresas con pequeñas pérdidas versus empresas con pequeños beneficios (Activo) } \\
\hline RN & \multicolumn{3}{|c|}{ Pequeñas pérdidas } & RN & \multicolumn{3}{|c|}{ Pequeños beneficios } & \multicolumn{3}{|c|}{ Test-diferencias } \\
\hline Intervalo & $\mathrm{N}$ & media & mediana & desv & Intervalo & $\mathrm{N}$ & media & mediana & desv & media & mediana \\
\hline$-0,0082<\mathrm{RN}<0$ & 228 & 31.519 & 4.360 & 187.802 & $0<\mathrm{RN}<0,0082$ & 669 & 8.824 & 2.778 & 21.868 & 0,0700 & 0,0001 \\
\hline$-0,016<\mathrm{RN}<-0,0082$ & 206 & 22.242 & 4.617 & 94.726 & $0,0082<\mathrm{RN}<0,016$ & 589 & 8.327 & 2.359 & 20.437 & 0,0377 & 0,0000 \\
\hline$-0,025<\mathrm{RN}<-0,016$ & 167 & 14.806 & 4.127 & 45.619 & $0,016<\mathrm{RN}<0,025$ & 541 & 8.865 & 2.386 & 40.893 & 0,1000 & 0,0000 \\
\hline$-0,033<\mathrm{RN}<-0,025$ & 106 & 9.874 & 3.788 & 24.335 & $0,025<\mathrm{RN}<0,033$ & 527 & 12.319 & 2.304 & 75.249 & 0,7408 & 0,0302 \\
\hline$-0,041<\mathrm{RN}<-0,033$ & 83 & 10.013 & 3.333 & 19.848 & $0,033<\mathrm{RN}<0,041$ & 457 & 8.381 & 2.051 & 34.096 & 0,6725 & 0,0014 \\
\hline
\end{tabular}

\begin{tabular}{|c|c|c|c|c|c|c|c|c|c|c|c|}
\hline \multicolumn{7}{|c|}{ Panel C: Empresas con pequeñas pérdidas versus empresas con pequeños beneficios (Ventas) } \\
\hline RN & \multicolumn{3}{|c|}{ Pequeñas pérdidas } & \multicolumn{2}{|c|}{ RN } & \multicolumn{3}{|c|}{ Pequeños beneficios } & \multicolumn{3}{|c|}{ Test-diferencias } \\
\hline Intervalo & $\mathrm{N}$ & media & mediana & desv & Intervalo & $\mathrm{N}$ & media & mediana & desv & media & mediana \\
\hline$-0,0082<\mathrm{RN}<0$ & 219 & 8.156 & 1.505 & 52.472 & $0<\mathrm{RN}<0,0082$ & 662 & 3.183 & 1.507 & 5.118 & 0,1628 & 0,9209 \\
\hline$-0,016<\mathrm{RN}<-0,0082$ & 198 & 4.220 & 1.656 & 11.416 & $0,0082<\mathrm{RN}<0,016$ & 586 & 3.050 & 1.381 & 5.075 & 0,1641 & 0,2424 \\
\hline$-0,025<\mathrm{RN}<-0,016$ & 159 & 3.126 & 1.398 & 4.455 & $0,016<\mathrm{RN}<0,025$ & 539 & 3.255 & 1.491 & 8.305 & 0,8503 & 0,5103 \\
\hline$-0,033<\mathrm{RN}<-0,025$ & 103 & 3.834 & 1.344 & 7.622 & $0,025<\mathrm{RN}<0,033$ & 526 & 4.671 & 1.496 & 22.794 & 0,7128 & 0,7590 \\
\hline$-0,041<\mathrm{RN}<-0,033$ & 79 & 3.369 & 1.711 & 5.978 & $0,033<\mathrm{RN}<0,041$ & 456 & 3.995 & 1.483 & 16.925 & 0,7452 & 0,3472 \\
\hline
\end{tabular}


Cuadro A-1.-Análisis del denominador de los resultados: Activo $\left(\mathrm{AT}_{\mathrm{t}-1}\right)$ (cont.)

\begin{tabular}{|c|c|c|c|c|c|c|c|c|c|c|c|}
\hline \multicolumn{7}{|c|}{ Panel D: Empresas con pérdidas elevadas versus empresas con beneficios elevados (Activo) } \\
\hline RN & \multicolumn{3}{|c|}{ Elevadas pérdidas } & \multicolumn{2}{|c|}{ RN } & \multicolumn{3}{|c|}{ Elevados beneficios } & \multicolumn{3}{|c|}{ Test-diferencias } \\
\hline Interval0 & $\mathrm{N}$ & media & mediana & desv & intervalo & $\mathrm{N}$ & media & medians & desv & media & mediana \\
\hline RN<-1,00 & 9 & 749 & 312 & 816 & $\mathrm{RN}>1,00$ & 11 & 4.247 & 85 & 9.359 & 0,2449 & 0,0027 \\
\hline$-1,00<\mathrm{RN}<-0,500$ & 19 & 813 & 372 & 1.121 & $0,500<\mathrm{RN}<1,00$ & 52 & 1.996 & 649 & 5.137 & 0,3251 & 0,1145 \\
\hline$-0,500<\mathrm{RN}<-0,400$ & 5 & 699 & 286 & 967 & $0,400<\mathrm{RN}<1,00$ & 43 & 1.925 & 654 & 3.035 & 0,3780 & 0,2322 \\
\hline
\end{tabular}

\begin{tabular}{|c|c|c|c|c|c|c|c|c|c|c|c|}
\hline \multicolumn{7}{|c|}{ Panel E: Empresas con pérdidas elevadas versus empresas con beneficios elevados (Ventas) } \\
\hline RN & \multicolumn{3}{|c|}{ Elevadas pérdidas } & \multicolumn{2}{c|}{ RN } & \multicolumn{3}{|c|}{ Elevados beneficios } & \multicolumn{3}{c|}{ Test-diferencias } \\
\hline Intervalo & $\mathrm{N}$ & media & medians & desv & Interval0 & $\mathrm{N}$ & media & medians & deav & media & medians \\
\hline $\mathrm{RN}<-1,00$ & 8 & 2.424 & 605 & 3.017 & $\mathrm{RN}>1,00$ & 9 & 3.194 & 499 & 5.684 & 0,7371 & 0,8884 \\
\hline$-1,00<\mathrm{RN}<-0,500$ & 16 & 1.317 & 1.139 & 893 & $0,500<\mathrm{RN}<1,00$ & 51 & 2.146 & 1.077 & 3.031 & 0,0888 & 0,8831 \\
\hline$-0,500<\mathrm{RN}<-0,400$ & 5 & 1.108 & 545 & 968 & $0,400<\mathrm{RN}<1,00$ & 43 & 3.335 & 1.514 & 4.715 & 0,3019 & 0,0993 \\
\hline
\end{tabular}

\begin{tabular}{|c|c|c|c|c|c|c|c|c|c|c|c|}
\hline \multicolumn{7}{|c|}{ Panel F: Empresas con elevadas pérdidas versus empresas con pequeñas pérdidas (Activo) } \\
\hline RN & \multicolumn{3}{|c|}{ Elevadas pérdidas } & \multicolumn{2}{|c|}{ RN } & \multicolumn{3}{|c|}{ Pequeñas pérdidas } & \multicolumn{3}{|c|}{ Test-diferencias } \\
\hline Intervalo & $\mathrm{N}$ & media & mediana & desv & Intervalo & $\mathrm{N}$ & media & medians & desv & media & mediana \\
\hline RN<-1,00 & 9 & 749 & 312 & 816 & $-0,0082<\mathrm{RN}<0$ & 228 & 31.519 & 4.360 & 187.802 & 0,6242 & 0,9072 \\
\hline$-1,00<\mathrm{RN}<-0,500$ & 19 & 813 & 372 & 1.121 & $-0,016<\mathrm{RN}<-0,0082$ & 206 & 22.242 & 4.617 & 94.726 & 0,3261 & 0,0000 \\
\hline$-0,500<\mathrm{RN}<-0,400$ & 5 & 699 & 286 & 967 & $-0,025<\mathrm{RN}<-0,016$ & 167 & 14.806 & 4.127 & 45.619 & 0,2288 & 0,9056 \\
\hline
\end{tabular}

\begin{tabular}{|c|c|c|c|c|c|c|c|c|c|c|c|}
\hline \multicolumn{7}{|c|}{ Panel G: Empresas con elevadas pérdidas versus empresas con pequeñas pérdidas (Ventas) } \\
\hline RN & \multicolumn{7}{|c|}{ Elevadas pérdidas } & \multicolumn{2}{|c|}{ RN } & \multicolumn{3}{|c|}{ Pequeñas pérdidas } & \multicolumn{3}{c|}{ Test-diferencias } \\
\hline Interval0 & $\mathrm{N}$ & media & medians & desv & Interval0 & $\mathrm{N}$ & media & medisna & desv & media & medisna \\
\hline RN<-1,00 & 8 & 2.424 & 605 & 3.017 & $-0,0082<\mathrm{RN}<0$ & 219 & 8.156 & 1.505 & 52.472 & 0,7581 & 0,2430 \\
\hline$-1,00<\mathrm{RN}<-0,500$ & 16 & 1.317 & 1.139 & 893 & $-0,016<\mathrm{RN}<-0,0082$ & 198 & 4.220 & 1.656 & 11.416 & 0,3115 & 0,0478 \\
\hline$-0,500<\mathrm{RN}<-0,400$ & 5 & 1.108 & 545 & 968 & $-0,025<\mathrm{RN}<-0,016$ & 159 & 3.126 & 1.398 & 4.455 & 0,1174 & 0,0183 \\
\hline
\end{tabular}


Cuadro A-1.-Análisis del denominador de los resultados: Activo ( $\left.\mathrm{AT}_{\mathrm{t}-1}\right)$ (cont.)

\begin{tabular}{|c|c|c|c|c|c|c|c|c|c|c|c|}
\hline \multicolumn{7}{|c|}{ Panel H: Empresas con elevados beneficios versus empresas con pequeños beneficios (Activo) } \\
\hline RN & \multicolumn{3}{|c|}{ Elevados beneficios } & \multicolumn{3}{|c|}{ RN } & \multicolumn{3}{|c|}{ Pequeños beneficios } & \multicolumn{3}{c|}{ Test-diferencias } \\
\hline Intervalo & $\mathrm{N}$ & media & mediana & desv & Intervalo & $\mathrm{N}$ & media & mediana & desv & media & mediana \\
\hline RN $>1,00$ & 11 & 4.247 & 85 & 9.359 & $0<\mathrm{RN}<0,0082$ & 669 & 8.824 & 2.778 & 21.868 & 0,4887 & 0,0033 \\
\hline $0,500<\mathrm{RN}<1,00$ & 52 & 1.996 & 649 & 5.137 & $0,0082<\mathrm{RN}<0,016$ & 589 & 8.327 & 2.359 & 20.437 & 0,2190 & 0,0047 \\
\hline $0,400<\mathrm{RN}<1,00$ & 43 & 1.925 & 654 & 3.035 & $0,016<\mathrm{RN}<0,025$ & 541 & 8.865 & 2.386 & 40.893 & 0,2667 & 0,9959 \\
\hline
\end{tabular}

\begin{tabular}{|c|c|c|c|c|c|c|c|c|c|c|c|}
\hline \multicolumn{7}{|c|}{ Panel I: Empresas con elevados beneficios versus empresas con pequeños beneficios (Ventas) } \\
\hline RN & \multicolumn{3}{|c|}{ Elevados beneficios } & RN & \multicolumn{3}{|c|}{ Pequeños beneficios } & \multicolumn{3}{|c|}{ Test-diferencias } \\
\hline Interval0 & $\mathrm{N}$ & media & medians & desv & Interval0 & $\mathrm{N}$ & media & mediana & desv & media & medians \\
\hline RN>1,00 & 9 & 3.194 & 499 & 5.684 & $0<\mathrm{RN}<0,0082$ & 662 & 3.183 & 1.507 & 5.118 & 0,9947 & 0,0264 \\
\hline $0,500<\mathrm{RN}<1,00$ & 51 & 2.146 & 1.077 & 3.031 & $0,0082<\mathrm{RN}<0,016$ & 586 & 3.050 & 1.381 & 5.075 & 0,9947 & 0,0264 \\
\hline $0,400<\mathrm{RN}<1,00$ & 43 & 3.335 & 1.514 & 4.715 & $0,016<\mathrm{RN}<0,025$ & 539 & 3.255 & 1.491 & 8.305 & 0,9506 & 0,9951 \\
\hline
\end{tabular}

